\title{
Products of Beta matrices and sticky flows
}

\author{
Y. Le Jan, S. Lemaire \\ March 2004 \\ Université Paris-Sud \\ Laboratoire de Mathématique \\ Bâtiment 425 \\ 91405 Orsay cedex \\ Yves.LeJan@math.u-psud.fr \\ Sophie.Lemaire@math.u-psud.fr
}

\begin{abstract}
A discrete model of Brownian sticky flows on the unit circle is described: it is constructed with products of Beta matrices on the discrete torus. Sticky flows are defined by their "moments" which are consistent systems of transition kernels on the unit circle. Similarly, the moments of the discrete model form a consistent system of transition matrices on the discrete torus. A convergence of Beta matrices to sticky kernels is shown at the level of the moments. As the generators of the n-point processes are defined in terms of Dirichlet forms, the proof is performed at the level of the Dirichlet forms. The evolution of a probability measure by the flow of Beta matrices is described by a measure-valued Markov process. A convergence result of its finite dimensional distributions is deduced.
\end{abstract}

Keywords : Markov chains with continuous parameter, Polya urns, Dirichlet laws, stochastic flow of kernels, Feller semigroups, Dirichlet forms, convergence of resolvents.

2000 MSC classifications : 60J27, 60J35, 60G09

\section{Introduction}

In [5, 4], a family of stochastic flows of kernels on the circle $S^{1}$ called "sticky flows" is described. Sticky flows interpolate between Arratia's flow of coalescing maps and the deterministic heat flow. They are defined by their "moments" which are consistent systems of transition kernels on $S^{1}$. In this article, a discrete version of sticky flows is presented for sticky flows associated with Brownian motions on $S^{1}$. This discrete model is defined by products of Beta matrices on the discrete torus $\frac{1}{N}(\mathbb{Z} / N \mathbb{Z})$. It appears to be a special case of a general construction which associates a flow of Dirichlet matrices to any Markov chain on a finite set. 
As in the continuous case, the moments of the flow of Beta matrices are consistent systems of transition matrices on $\frac{1}{N}(\mathbb{Z} / N \mathbb{Z})$. The convergence of the flow of Beta matrices towards sticky kernels is shown at the level of the moments. Namely, it is established that for every $n \in \mathbb{N}^{*}$, the semigroup $\left\{P_{t}^{(n)}, t \in \mathbb{R}\right\}$ of the $n$-point motion of the sticky flow is approximated by the $n$-point transition matrix of the flow of Beta matrices. Classical approximation theorems, such as theorem 6.1 in [1], cannot be used since the generators of the semigroups $P_{t}^{(n)}$ do not have a core of $C^{2}$ functions. The generators are defined in terms of Dirichlet forms, thus the proof is performed at the level of the Dirichlet forms. Given an initial law, the flow of Beta matrices generates a measure-valued Markov process. A convergence result of its finite dimensional distributions is deduced.

Section 1 contains a study of the flows of Dirichlet matrices on a finite set. Flows of Beta matrices on $\frac{1}{N}(\mathbb{Z} / N \mathbb{Z})$ and Brownian sticky flows on the circle $S^{1}$ are presented in section 2. Section 3 is devoted to establishing the convergence of the flow of Beta matrices on $\frac{1}{N}(\mathbb{Z} / N \mathbb{Z})$ to a Brownian sticky flow on $S^{1}$.

\section{Dirichlet matrices and Polya scheme}

A stochastic kernel on a finite set $F$ is nothing but a random transition matrix on $F$. Given a sequence of i.i.d. random transition matrices $\left(K_{i}\right)_{i \in \mathbb{Z}}$ on $F$, one may define a stochastic flow of kernels $\left(K_{s, t}\right)_{s \leq t}$ on $F$ by setting for every $s \leq t$,

$$
K_{s, t}= \begin{cases}K_{s+1} \ldots K_{t-1} K_{t} & \text { if the time is } \mathbb{Z} \\ K_{Z(s)+1} \ldots K_{Z(t)-1} K_{Z(t)} & \text { if the time is } \mathbb{R} .\end{cases}
$$

where $Z$ denotes a homogeneous Poisson process independent of $\left(K_{i}\right)_{i}$.

The sequence of matrices $\left(K_{i}\right)_{i}$ defines a random medium that evolves with time. It is also a random distribution on paths. The $n$-point motion is defined as an $n$-sample of this distribution. More precisely, the environment (i.e. the sequence $\left.\left(K_{i}\right)_{i}\right)$ being fixed, one considers the motion of $n$ independent inhomogeneous Markov chains with transition kernels $\left(K_{i}\right)_{i}$. Under the annealed measure (i.e. averaging on the environment), the motion of $n$ points becomes a homogeneous Markov chain with semigroup $\left\{P_{t}^{(n)}:=E\left(K_{0, t}^{\otimes n}\right)\right\}$. The semigroups $\left(P_{t}^{(n)}\right)_{t}, n \in \mathbb{N}^{*}$ form a consistent exchangeable family of Markovian semigroups: all points play the same role and by removing any point in the $n$-point motion, one gets the $(n-1)$-point motion. In [3], following a generalization of De Finetti theorem, it is shown that the law of such a stochastic flow of kernels is given by a consistent family of $n$-point Markovian semigroups $\left\{P_{t}^{(n)}, n \in \mathbb{N}^{*}\right\}$.

\subsection{Dirichlet matrices}

We shall assume that the rows of every matrix $K_{i}$ are independent Dirichlet random vectors. A suitable choice of the coefficients of the Dirichlet laws enables us to exhibit a consistent and exchangeable system of probability measures $\left\{m^{(n)}, n \in \mathbb{N}^{*}\right\}$ such that for every $n \in \mathbb{N}^{*}, m^{(n)}$ is an invariant measure for the $n$-point semigroup $\left(P_{t}^{(n)}\right)_{t}$.

Let us first state a natural extension of the Dirichlet distribution to the case of nonnegative coefficients and then define what we shall call a Dirichlet random matrix and a flow of Dirichlet matrices. 
Definition. Let $\alpha_{1}, \ldots, \alpha_{k}$ be nonnegative reals such that at least one of them are positive. Let $1 \leq i_{1}<\ldots<i_{j} \leq k$ denote the indices of the positive coefficients and $V$ the set of points $x=\left(x_{1}, \ldots, x_{k}\right) \in[0,1]^{k}$ such that $x_{i}=0$ if $i \notin\left\{i_{1}, \ldots, i_{j}\right\}$ and $\sum_{i=1}^{k} x_{i}=1$.

A random vector $X=\left(X_{1}, \ldots, X_{k}\right)$ is said to have the Dirichlet distribution $\mathcal{D}\left(\alpha_{1}, \ldots, \alpha_{k}\right)$ if $P(X \notin V)=0$ and if $\left(X_{i_{1}}, \ldots, X_{i_{j}}\right)$ has the Dirichlet law $\mathcal{D}\left(\alpha_{i_{1}}, \ldots, \alpha_{i_{j}}\right)$ i.e.

$$
\frac{\Gamma\left(\alpha_{i_{1}}+\cdots+\alpha_{i_{j}}\right)}{\Gamma\left(\alpha_{i_{1}}\right) \cdots \Gamma\left(\alpha_{i_{j}}\right)} x_{1}^{\alpha_{i_{1}}-1} \cdots x_{j}^{\alpha_{i_{j}}-1} \mathbf{1}_{\left\{x_{1}+\cdots+x_{j}=1\right\}} d x_{1} \cdots d x_{j-1} .
$$

Definition. Let $F$ and $G$ be two finite subsets. Let $A=\left(a_{i, j}\right)_{(i, j) \in F \times G}$ be a matrix of nonnegative coefficients such that each row has at least a positive coefficient.

A random matrix $X=\left(X_{i, j}\right)_{(i, j) \in F \times G}$ will be called a Dirichlet matrix with parameter $A$ if the rows of $X$ are independent random vectors and the $i$-th row of $X$ has the Dirichlet distribution whose parameters are given by the $i$-th row of $A$ for every $i \in F$.

Definition. Let $F$ be a finite set and $A=\left(a_{i, j}\right)_{(i, j) \in F^{2}}$ be a matrix of nonnegative coefficients such that each row has at least a positive coefficient.

The discrete time (or continuous time) stochastic flows defined by formula (11) from a family $\left(K_{i}\right)_{i \in \mathbb{Z}}$ of independent Dirichlet matrices of parameter $A$ is said to be a stochastic flow of Dirichlet matrices of parameter $A$ on $F$.

Let us notice that the product of two independent Dirichlet matrices is a matrix whose rows are generally neither independent nor distributed according to a Dirichlet distribution. Still, the following lemma holds; it states a probably well-known property of Dirichlet laws which is based on properties of Gamma distributions (first?) mentioned by Edwin Pitman in [6].

Lemma 1 Let $r$ and $s$ be positive integers. Let $A=\left(a_{i, j}\right)_{1 \leq i \leq r, 1 \leq j \leq s}$ be a matrix of nonnegative coefficients such that each row has at least a positive coefficient. Let $X$ be a Dirichlet matrix of parameter A.

If $Y$ is a random vector independent of the random matrix $X$, with Dirichlet distribution $\mathcal{D}\left(\sum_{j=1}^{s} a_{1, j}, \ldots, \sum_{j=1}^{s} a_{r, j}\right)$, then the random vector $Y X$ has the Dirichlet distribution $\mathcal{D}\left(\sum_{i=1}^{r} a_{i, 1}, \ldots, \sum_{i=1}^{r} a_{i, s}\right)$.

Proof. We shall construct copies of $X$ and $Y$ using a family of independent Gamma random variables. This construction is based on the following properties of the Gamma laws:

Properties 2 Let $\alpha_{1}, \ldots, \alpha_{r}$ be nonnegative reals and $Z_{1}, \ldots, Z_{r}$ be a family of independent random variables with Gamma laws $\operatorname{Gamma}\left(\alpha_{1}, 1\right), \ldots, \operatorname{Gamma}\left(\alpha_{r}, 1\right)$ respectively (by convention, the Gamma law of parameter 0 is the Dirac at 0$)$. Assume that some of the parameters $\alpha_{1}, \ldots, \alpha_{r}$ are positive. Then

- the random variable $Z=\sum_{i=1}^{r} Z_{i}$ follows the law Gamma $\left(\sum_{i=1}^{r} \alpha_{i}, 1\right)$.

- the random vector $\left(\frac{Z_{1}}{Z}, \ldots, \frac{Z_{r}}{Z}\right)$ has the Dirichlet law $\mathcal{D}\left(\alpha_{1}, \ldots, \alpha_{r}\right)$ and is independent of the random variable $Z$. 
These properties can be shown by computing the Laplace transform of the random vector $\left(\frac{Z_{1}}{Z}, \ldots, \frac{Z_{r}}{Z}, Z\right)$.

Let $\left\{U_{i, j},(i, j) \in\{1, \ldots, r\} \times\{1, \ldots, s\}\right\}$ denote a family of independent random variables such that $U_{i, j}=0$ if $a_{i, j}=0$ and $U_{i, j}$ has the $\operatorname{Gamma}\left(a_{i, j}, 1\right)$ distribution if $a_{i, j}>0$. Set $U_{i}=\sum_{j=1}^{s} U_{i, j}, U=\sum_{i=1}^{r} U_{i}$ and $V_{i, j}=\frac{U_{i, j}}{U_{i}}$ for every $(i, j) \in\{1, \ldots, r\} \times$ $\{1, \ldots, s\}$. It follows from the recalled properties of the Gamma laws that for every $i \in\{1, \ldots, r\}$, the random vector $V_{i}=\left(V_{i, 1}, \ldots, V_{i, s}\right)$ has the Dirichlet distribution $\mathcal{D}\left(a_{i, 1}, \ldots, a_{i, s}\right)$ and is independent of $U_{i}$ that has the $\operatorname{Gamma}\left(\sum_{j=1}^{s} a_{i, j}, 1\right)$ distribution. As $\left\{U_{i, j},(i, j) \in\{1, \ldots, r\} \times\{1, \ldots, s\}\right\}$ is a family of independent random variables, the random variables $V_{1}, \ldots, V_{r}, U_{1}, \ldots, U_{r}$ are independent. In particular, $V=\left(V_{i, j}\right)_{i, j}$ is a Dirichlet matrix of parameter $A$.

It also follows that the random vector $W=\left(\frac{U_{1}}{U}, \ldots, \frac{U_{r}}{U}\right)$ has the same law as $Y$, i.e. the Dirichlet distribution $\mathcal{D}\left(\sum_{j=1}^{s} a_{1, j}, \ldots, \sum_{j=1}^{s} a_{r, j}\right)$ and is independent of the random vectors $V_{1}, \ldots, V_{r}$.

If we set $T_{j}=\sum_{i=1}^{r} W_{i} V_{i, j}$ for $j \in\{1, \ldots, s\}$, then $T_{j}=\frac{1}{U} \sum_{i=1}^{r} U_{i, j}$. As the random variables $\sum_{i=1}^{r} U_{i, j}$ for $j \in\{1, \ldots, s\}$ are independent and have the $\operatorname{Gamma}\left(\sum_{i=1}^{r} a_{i, 1}, 1\right), \ldots$, $\operatorname{Gamma}\left(\sum_{i=1}^{r} a_{i, s}, 1\right)$ distributions respectively, the random vector $\left(T_{1}, \ldots, T_{s}\right)$ has the Dirichlet distribution $\mathcal{D}\left(\sum_{i=1}^{r} a_{i, 1}, \ldots, \sum_{i=1}^{r} a_{i, s}\right)$.

\section{$2.2 \quad$ Invariant distributions}

Let us notice that if $P$ is a stochastic matrix indexed by $F$ and $m$ is a finite positive measure on $F$ invariant by $P$ then the matrix $A=\left(a_{i, j}\right)$ defined by $a_{i, j}=m_{i} P_{i, j}$ for every $(i, j) \in F^{2}$ is such that the sum of the coefficients of the $i$-th row is equal to the sum of the coefficients of the $i$-th column for every $i \in F$. Thus applying lemma 1 yields:

Proposition 3 Let $P$ be a stochastic matrix indexed by a finite set $F$ and let $m$ be $a$ positive measure on $F$ invariant by $P$. Set $A=\left(a_{i, j}\right)$ the matrix defined by $a_{i, j}=m_{i} p_{i, j}$ for every $(i, j) \in F^{2}$. Let $\left(K_{s, t}\right)_{s \leq t}$ be a discrete time stochastic flow of Dirichlet matrices of parameter $A$. Let $\mu$ be a Dirichlet vector on $F$ of parameter $m$, independent of $\left(K_{s, t}\right)_{s \leq t}$.

(i) For every $s \leq t, \mu K_{s, t}$ is a Dirichlet vector of parameter $m$.

(ii) For every $n \in \mathbb{N}^{*}, E\left(\mu^{\otimes n}\right)$ is an invariant probability measure for the $n$-point semigroup $\left(P_{t}^{(n)}\right)_{t}$ associated with the Dirichlet flow $\left(K_{s, t}\right)_{s \leq t}$. Its expression can be given iteratively as follows:

$$
E\left(\mu^{\otimes n}\right)(x)=\frac{m\left(x_{n}\right)+\sum_{i=1}^{n-1} \mathbf{1}_{\left\{x_{n}=x_{i}\right\}}}{m(F)+n-1} E\left(\mu^{\otimes(n-1)}\right)(\underline{x}) .
$$

for every $x=\left(\underline{x}, x_{n}\right) \in F^{n}$.

(iii) Assume that $P$ is periodic with period $d>1$. There exists a partition $C_{0}, \ldots, C_{d-1}$ of $F$ such that $p_{i, j}>0$ only if there exists $r \in\{0, \ldots, d-1\}$ such that $i \in C_{r}$ and $j \in C_{r+1}$ (by convention $C_{j d+k}=C_{k}$ for every $j \in \mathbb{N}$ and $k \in\{0, \ldots, d-1\}$ ). Then for every $k, r \in\{0, \ldots, d-1\}$ and $j \in \mathbb{N}, \mu_{\mid C_{k}} K_{0, d j+r}$ is a Dirichlet vector on $F$ of parameter $m \mathbf{1}_{C_{k+r}}$. 
Proof. Assertion ( $i$ ) follows from lemma 1 For every $n \in \mathbb{N}^{*}$ and $t \in \mathbb{N}, E\left(\left(\mu K_{0, t}\right)^{\otimes n}\right)$ is equal to $E\left(\mu^{\otimes n}\right) P_{t}^{(n)}$, thus $E\left(\mu^{\otimes n}\right)$ is invariant by $P_{t}^{(n)}$.

Let us compute the moments of $\mu$. Set $e_{1}, \ldots, e_{r}$ the elements of $F$. For $x \in F^{n}$, let $s_{e}(x)$ denote the number of coordinates of $x$ that equal to $e$. As $E\left(\mu^{\otimes n}\right)(x)$ is the moment of order $\left(s_{e_{1}}(x), \ldots, s_{e_{r}}(x)\right)$ of the Dirichlet law $\mathcal{D}\left(m\left(e_{1}\right), \ldots, m\left(e_{r}\right)\right)$,

$$
\begin{aligned}
E\left(\mu^{\otimes n}\right)(x) & =\frac{\Gamma(m(F))}{\Gamma(m(F)+n)} \prod_{e \in F} \frac{\Gamma\left(m(e)+s_{e}(x)\right)}{\Gamma(m(e))} \\
& =\frac{1}{\gamma_{m(F)}(n)} \prod_{e \in F} \gamma_{m(e)}\left(s_{e}(x)\right) .
\end{aligned}
$$

where $\gamma_{a}(u)$ denotes the product $\prod_{i=0}^{u-1}(a+i)$ for every $u \in \mathbb{N}^{*}$ and $a \in \mathbb{R}$. To obtain the iterative expression, it remains to notice that if $x=\left(\underline{x}, x_{n}\right)$ then $s_{e}(x)=\mathbf{1}_{\left\{x_{n}=e\right\}}+s_{e}(\underline{x})$. The restriction of $\mu$ to $C_{k}$ is the random measure $\mu_{\mid C_{k}}(\cdot)=\frac{\mu\left(C_{k} \cap \cdot\right)}{\mu\left(C_{k}\right)}$. As $\mu$ is a Dirichlet vector of parameter $m, \mu_{\mid C_{k}}$ is a Dirichlet vector of parameter $m \mathbf{1}_{C_{k}}$. Lemma 1 applies to the vector $\left(\mu_{\mid C_{k}}(i)\right)_{i \in C_{k}}$ and the submatrix $\tilde{K}_{l}=\left(K_{l, l+1}(i, j)\right)_{i \in C_{k}, j \in C_{k+1}}$ of $K_{l, l+1}$ for every $l \in \mathbb{Z}$; this shows that $\mu_{\mid C_{k}} K_{l, l+1}$ is a Dirichlet vector of parameter $m \mathbf{1}_{C_{k+1}}$ for every $k \in\{0, \ldots, d\}$ and $l \in \mathbb{Z}$.

In order to present an explicit expression of $E\left(\mu^{\otimes n}\right)$, let us introduce some notations associated with a partition $\pi$ of $[n]=\{1, \ldots, n\}$.

Let $\mathcal{P}_{n}$ be the set of all partitions of $[n]$. Let $|\pi|$ denote the number of non-empty blocks of $\pi$. The symbol $\preccurlyeq$ will be used to designate an order relation between partitions: $\pi^{\prime} \preccurlyeq \pi$ if $\pi^{\prime}$ is equal to $\pi$ or is a finer partition than $\pi$. Let $C_{\pi}$ be the set of points $x \in F^{n}$ such that $x_{i}=x_{j}$ if and only if $i$ and $j$ are in the same block of $\pi$. Set $E_{\pi}=\cup_{\pi^{\prime}, \pi \preccurlyeq \pi^{\prime}} C_{\pi^{\prime}}$. It is the set of points $x \in F^{n}$ such that, if $i$ and $j$ are in the same block of $\pi$, then $x_{i}=x_{j}$. It is isomorphic to $F^{|\pi|}$. Let $\phi_{\pi}: F^{|\pi|} \mapsto E_{\pi}$ be the one-to-one mapping defined as follows: for all $x \in F^{|\pi|}, \phi_{\pi}(x)=\left(y_{1}, \ldots, y_{n}\right)$ where $y_{i}=x_{l}$ if $i$ belongs to the $l$-th block of $\pi$. The measure $E\left(\mu^{\otimes n}\right)$ can be expressed as a combination of probability measures on the sets $E_{\pi}, \pi \in \mathcal{P}_{n}$ as follows:

Proposition 4 Let $m$ be a measure on a finite set $F$ and let $\mu$ be a Dirichlet vector on $F$ with parameter $m$. Set $\tilde{m}=\frac{m}{m(F)}$. For every $n \in \mathbb{N}^{*}$,

$$
E\left(\mu^{\otimes n}\right)=\sum_{\pi \in \mathcal{P}_{n}} p_{\pi}^{(m(F))} \phi_{\pi}\left(\tilde{m}^{\otimes|\pi|}\right)
$$

where $p_{\pi}^{(a)}=a^{k} \frac{\prod_{i=1}^{k}\left(n_{i}-1\right) !}{\prod_{i=0}^{n-1}(a+i)}$ if $\pi$ is a partition of $[n]$ with $k$ nonempty blocks of length $n_{1}, \ldots, n_{k}$ and $a$ is a positive real.

Proof. The proof can be deduced from the expression (2) of $E\left(\mu^{\otimes n}\right)$, by induction on $n$. Assume that formula (3) is true for $E\left(\mu^{\otimes(n-1)}\right)$. Fix a point $x=\left(\underline{x}, x_{n}\right) \in F^{n}$. If $\pi$ is the partition of $[n-1]$ such that $\underline{x} \in C_{\pi}$, then

$$
E\left(\mu^{\otimes n}\right)(x)=\sum_{\pi^{\prime}, \pi^{\prime} \preccurlyeq \pi} p_{\pi^{\prime}}^{(m(F))} \phi_{\pi^{\prime}}\left(\tilde{m}^{\otimes\left|\pi^{\prime}\right|}\right)(\underline{x}) \frac{m\left(x_{n}\right)+\sum_{i=1}^{n-1} \mathbf{1}_{\left\{x_{i}=x_{n}\right\}}}{m(F)+n-1} .
$$


Set $B_{1}, \ldots, B_{k}$ denote the nonempty blocks of the partition $\pi$. Let $s(\pi)$ denote the partition $(\pi,\{n\})$ and $s_{i}(\pi)$ denote the partition of $[n]$ obtained from $\pi$ by adding $n$ to the block $B_{i}$ for $i \in\{1, \ldots, k\}$. Two cases arise according as $x \in C_{s(\pi)}$ or $x \in C_{s_{i}(\pi)}$ for some $i \in\{1, \ldots, k\}$ since for every $a>0$,

$$
p_{s(\pi)}^{(a)}=\frac{a}{a+n-1} p_{\pi}^{(a)} \text { and } p_{s_{i}(\pi)}^{(a)}=\frac{\operatorname{Card}\left(B_{i}\right)}{a+n-1} p_{\pi}^{(a)} \text { for } i \in\{1, \ldots, k\} .
$$

Then formula (3) for $E\left(\mu^{\otimes n}\right)$ follows from the fact that:

- the partitions $\tilde{\pi}$ finer than $s(\pi)$ have the form $s\left(\pi^{\prime}\right)$ where $\pi^{\prime}$ is finer than $\pi$,

- the partitions finer than $s_{i}(\pi)$ either have the form $s\left(\pi^{\prime}\right)$ where $\pi^{\prime}$ is a partition of $[n-1]$ finer than $\pi$, or have the form $s_{j}\left(\pi^{\prime}\right)$ where $\pi^{\prime}$ is a partition of $[n-1]$ finer than $\pi$ such that its $j$-th block is a subset of $B_{i}$.

Remark. $\left(p_{\pi}^{(a)}\right)_{\pi \in \mathcal{P}_{n}}$ is the exchangeable partition function of an exchangeable sequence of random variables governed by the Blackwell-MacQueen Urn scheme [7.

\subsection{Description of the $n$-point motion}

In order to complete the description of the $n$-point motion associated with the discrete time flow of Dirichlet matrices of parameter $A$ denoted by $\left(K_{s, t}\right)_{s \leq t}$, let us compute the transition matrix $P^{(n)}$ : for $x=\left(x_{1}, \ldots, x_{n}\right)$ and $y=\left(y_{1}, \ldots, y_{n}\right)$ in $F^{n}$,

$$
P^{(n)}(x, y)=E\left(\prod_{i=1}^{n} K_{1}\left(x_{i}, y_{i}\right)\right)=\prod_{l \in F} E\left(\prod_{h \in F} K_{1}(l, h)^{s_{l, h}(x, y)}\right)
$$

where $s_{l, h}(x, y)=\operatorname{Card}\left(\left\{i,\left(x_{i}, y_{i}\right)=(l, h)\right\}\right)$. Let us set $\gamma_{a}(u)=\prod_{i=0}^{u-1}(a+i)$ and $s_{l}(x)=\operatorname{Card}\left(\left\{i \in\{1, \ldots, n\}, x_{i}=l\right\}\right)$. It follows from the expression of the moments of the Dirichlet laws that

$$
P^{(n)}(x, y)=\prod_{i \in F} \frac{\prod_{j \in F} \gamma_{a_{i, j}}\left(s_{i, j}(x, y)\right)}{\gamma_{\sum_{j \in F} a_{i, j}}\left(s_{i}(x)\right)}
$$

Let us remark that:

$$
P^{(1)}(x, y)=\frac{a_{x, y}}{\sum_{z \in F} a_{x, z}} \text { and } P^{(n)}(x, y)=\frac{a_{x_{n}, y_{n}}+s_{x_{n}, y_{n}}(\underline{x}, \underline{y})}{\sum_{j \in F} a_{x_{n}, j}+s_{x_{n}}(\underline{x})} P^{(n-1)}(\underline{x}, \underline{y})
$$

for $n \geq 2$ where $\underline{x}=\left(x_{1}, \ldots, x_{n-1}\right)$.

Thus the transition mechanism can be described as follows: the first point moves from a site $i$ to a site $j$ with probability $p_{i, j}=\frac{a_{i, j}}{\sum_{\ell \in F} a_{i, \ell}}$. The motion of the $(k-1)$ first points being known, the $k$-th point moves from a site $i$ to a site $j$ with probability $\frac{a_{i, j}+u}{\sum_{\ell \in F} a_{i, \ell}+v}$ if among the $k-1$ first points, $v$ were located on the site $i$, and $u$ have moved from $i$ to the site $j$. This is a combination of independent Polya urns attached at each site.

Let us deduce some elementary properties of $P^{(n)}$ : 
1. If the one-point motion is defined as a reversible Markov chain on $F$, then for every $n \in \mathbb{N}^{*}$ the $n$-point motion is a reversible Markov chain on $F^{n}$.

2. If $A$ is irreducible and aperiodic then for every $n \in \mathbb{N}^{*}, P^{(n)}$ is also an irreducible and aperiodic matrix.

3. Assume that $A$ is irreducible and periodic of period $d$. Let $C_{0}, \ldots, C_{d-1}$ denote a partition of $F$ such that for every $r \in\{0, \ldots, d-1\}, i \in C_{r}$ and $j \notin C_{r+1}$ imply $a_{i, j}=0$. Then for every $n \in \mathbb{N}^{*}$ and $r \in\{0, \ldots, d-1\},\left(C_{r}\right)^{n}$ is a closed subset for $P^{(n)}$ and $\left(P^{(n)}\right)^{d}$ is an irreducible aperiodic matrix on $\left(C_{r}\right)^{n}$.

The property (1) implies that if the one-point motion is a reversible Markov chain on $F$ with reversible probability measure $m$ and if $\mu$ is a Dirichlet vector on $F$ with parameter $m$ independent of the stochastic kernels $\left(K_{i}\right)_{i}$, then the stationary vector-valued Markov process $\left(\mu K_{0, j}\right)_{j}$ is reversible. The properties (2) and (3) of the $n$-point motions and proposition [3] enable us to establish the asymptotic behaviour of the flow of Dirichlet matrices:

Corollary 5 Let $P$ be a stochastic matrix indexed by a finite set $F$ and let $m$ be a positive measure on $F$ invariant by $P$. Set $A=\left(a_{i, j}\right)$ the matrix defined by $a_{i, j}=m_{i} p_{i, j}$ for every $(i, j) \in F^{2}$. Let $\left(K_{s, t}\right)_{s \leq t}$ be a discrete time stochastic flow of Dirichlet matrices of parameter $A$.

(i) Assume that $P$ is irreducible and aperiodic.

For every probability measure $\nu$ on $F, \nu K_{0, j}$ converges in law to a Dirichlet vector of parameter $m$ as $j$ tends to $+\infty$.

(ii) Assume that $P$ is irreducible and periodic of period d. Let $C_{0}, \ldots, C_{d-1}$ denote a partition of $F$ such that for every $r \in\{0, \ldots, d-1\}$, if $i \in C_{r}$ and $j \notin C_{r+1}$ then $p_{i, j}=0$. Set $C_{k+d}=C_{k}$ for every $k \in \mathbb{N}$. For $k \in\{0, \ldots, d-1\}$, let $\nu_{k}$ denote $a$ probability measure on $F$ such that $\nu_{k}\left(C_{k}\right)=1$.

For every $r$ in $\{0, \ldots, d-1\},\left(\nu_{0} K_{0, j d+r}, \ldots, \nu_{d-1} K_{0, j d+r}\right)$ converges in law, as $j$ tends to $+\infty$, to a vector $\left(\mu_{r}, \ldots, \mu_{r+d-1}\right)$ of independent Dirichlet vectors of parameters $m \mathbf{1}_{C_{r}}, \ldots, m \mathbf{1}_{C_{r+d-1}}$ respectively.

\section{Proof.}

(i) $\left(\nu K_{0, j}\right)_{j}$ is a sequence of random variables that take their values in the set of probability measures on $F$. To prove that they converge in law to a random measure $\mu$, it suffices to prove that for every $n \in \mathbb{N}^{*}$, the sequence of probability measures $\left(E\left(\left(\nu K_{0, j}\right)^{\otimes n}\right)\right)_{j}$ on $F^{n}$ converges to $E\left(\mu^{\otimes n}\right)$. As $P_{0, j}^{(n)}=E\left(K_{0, j}\right)^{\otimes n}$ is the transition matrix of an irreducible aperiodic Markov chain on $F^{n},\left(\nu^{\otimes n} P_{0, j}^{(n)}\right)_{j}$ converges to the stationary law of the Markov chain that is $E\left(\mu^{\otimes n}\right)$ where $\mu$ is a Dirichlet vector of parameter $m$, by proposition 3 .

(ii) Let us first note that for every $j \in \mathbb{N}, \nu_{0} K_{0, j}, \ldots, \nu_{d-1} K_{0, j}$ are independent variables since $K_{0, j}$ is a product of $j$ independent random matrices, each of them having independent rows. As $\left(P^{(n)}\right)^{d}$ is an irreducible and aperiodic Markov chain on $\left(C_{u}\right)^{n}$ for every $u \in\{0, \ldots, d-1\}$ and $n \in \mathbb{N}^{*}$, it can be shown as in the proof of $(i)$, that for every $k, r \in\{0, \ldots, d-1\}, \nu_{k} K_{0, d j+r}$ converges in law to a Dirichlet vector of parameter $m \mathbf{1}_{C_{k+r}}$ as $j$ tends to $+\infty$. 


\section{$3 \quad$ A discrete model of a sticky flow on $S^{1}$}

In the remaining part of this paper, we shall consider a particular flow of Dirichlet matrices on the lattice $T_{N}=\frac{1}{2 N}(\mathbb{Z} / 2 N \mathbb{Z})$ of $S^{1}$, called flow of Beta matrices. It gives a simple discrete model of the Brownian sticky flow defined by Y. Le Jan and O. Raimond in [5, 4. After a description of the flow of Beta matrices, the main properties of the Brownian sticky flows will be summarized.

\subsection{Beta matrices}

Let $a$ be a positive real, $N \in \mathbb{N}^{*}$ and $K$ be a matrix on the lattice $T_{N}$ defined as follows:

$$
K(i, j)=X_{i} \mathbf{1}_{\left\{j=i+\frac{1}{2 N}\right\}}+\left(1-X_{i}\right) \mathbf{1}_{\left\{j=i-\frac{1}{2 N}\right\}} \text { for every } i, j \in T_{N}
$$

where $X_{1}, \ldots, X_{2 N}$ are independent $\operatorname{Beta}\left(\frac{a}{2 N}, \frac{a}{2 N}\right)$ random variables.

The matrix $K$ is a Dirichlet matrix of parameter $A=\left(m_{i} p_{i, j}\right)_{i, j}$ where $P=\left(p_{i, j}\right)_{i, j}$ is the transition matrix of the symmetric random walk on $T_{N}$ and $m$ is the uniform measure on $T_{N}$ with total mass $2 a$. Let $\left(K_{i}\right)_{i \in \mathbb{Z}}$ be a sequence of independent matrices having the same law as $K$ and let $Z$ be an independent Poisson process on $\mathbb{R}$ with intensity $4 N^{2}$. The continuous time Dirichlet flow of matrices defined on $T_{N}$ by

$$
K_{N, s, t}=K_{Z(s)+1} K_{Z(s)+2} \cdots K_{Z(t)-1} K_{Z(t)} \text { for every } s \leq t,
$$

will be called a flow of Beta matrices on $T_{N}$ of parameter a. Let us note that $A$ is irreducible and two-periodic thus the $n$-point process is not irreducible. We shall focus our attention on the following irreducible set:

$$
\begin{aligned}
T_{N}^{(n)} & =\left\{\frac{x}{2 N}, x \in(\mathbb{Z} / 2 N \mathbb{Z})^{n} \text { and } x_{i}-x_{1} \in 2 \mathbb{Z}, \text { for all } 1 \leq i \leq n\right\} \\
& =\left(\frac{1}{N}(\mathbb{Z} / N \mathbb{Z})\right)^{n} \cup\left(\frac{1}{2 N}((2 \mathbb{Z}+1) / 2 N \mathbb{Z})\right)^{n}
\end{aligned}
$$

The transition matrix of the $n$-point motion on $T_{N}^{(n)}$ at time $t$ will be denoted $P_{N, t}^{(n)}$. The jump Markov chain of the $n$-point process is two-periodic: in one step, a point with odd coordinates moves to a point with even coordinates and conversely. Its transition matrix will be denoted $P_{N}^{(n)}$. It has a reversible probability measure denoted by $m_{N}^{(n)}$ :

$$
m_{N}^{(n)}=\frac{1}{2}\left(E\left(\mu_{N}^{(0) \otimes n}\right)+E\left(\mu_{N}^{(1) \otimes n}\right)\right)
$$

where $\mu_{N}^{(0)}$ and $\mu_{N}^{(1)}$ denote independent Dirichlet vectors on $T_{N}$ with parameter $m \mathbf{1}_{\frac{1}{N} \mathbb{Z} / N \mathbb{Z}}$ and $m \mathbf{1}_{\frac{1}{2 N}((2 \mathbb{Z}+1) / 2 N \mathbb{Z})}$ respectively. Let $\eta$ be a Bernoulli random variable with parameter $\frac{1}{2}$, independent of $\mu_{N}^{(0)}$ and $\mu_{N}^{(1)}$. It follows that if $\nu$ is a probability measure on $\frac{1}{N} \mathbb{Z} / N \mathbb{Z}$ or $\frac{1}{2 N}((2 \mathbb{Z}+1) / 2 N \mathbb{Z})$ then $\left(\nu K_{N, 0, t}\right)_{t \in \mathbb{R}_{+}}$converges in law to $\mu_{N}^{(\eta)}$ as $t$ tends to $+\infty$.

A sample path of the measured-valued Markov process $\nu^{(a)}=\left(\delta_{x} K_{N, 0,2 k}\right)_{k \in \mathbb{N}}$ associated to the discrete-time flow of Beta matrices on $\frac{1}{N}(\mathbb{Z} / N \mathbb{Z})$, is represented in figures 1] and 2 for $N=500, x=\frac{1}{2}$ and two choices of the parameter $a: a=20$ and $a=100$. In these figures, the sample of $\nu_{k}^{(a)}$ at a fixed $k$ is represented by a horizontal line of $N$ colored pixels. A detail of the histogram of the sample of $\nu_{k}^{(a)}$ for $k=500$ and $k=1000$ is given figures [5] and 6 . 

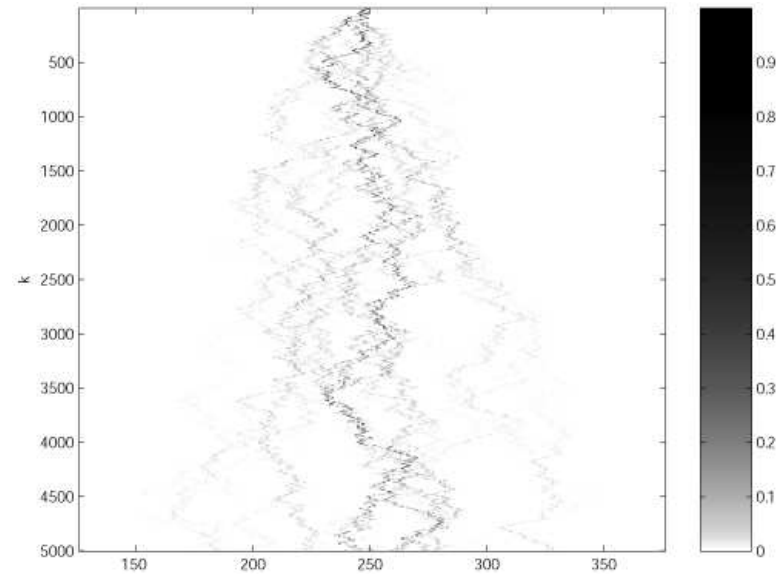

Figure 1: a sample of the measure-valued process $\left\{\nu_{k}^{(20)}, 0 \leq k \leq 5000\right\}$

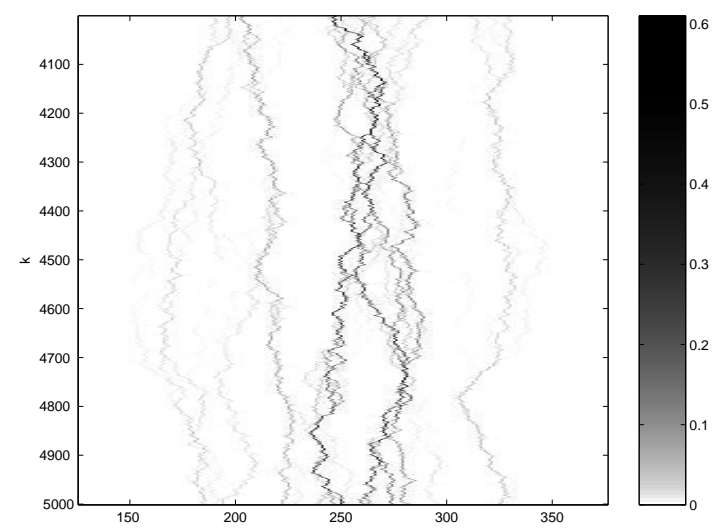

Figure 3: an enlargement of the bottom of figure 1
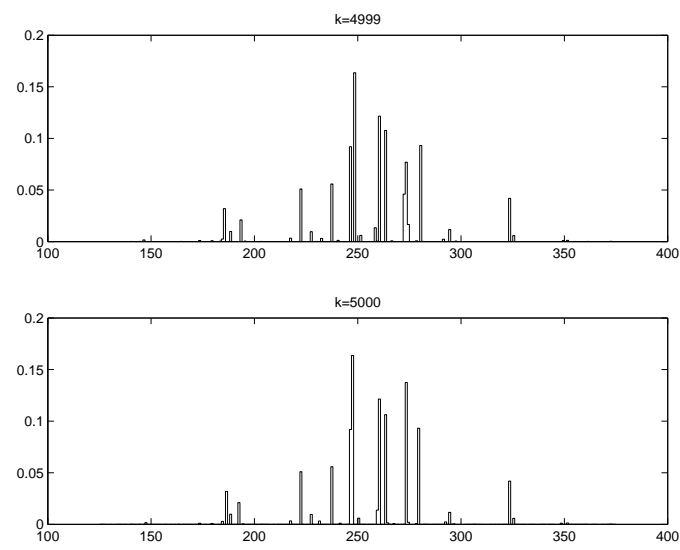

Figure 5: histograms of the sample of the probability measure $\nu_{k}^{(20)}$ for two successive values of $k: k=4999$ and $k=5000$

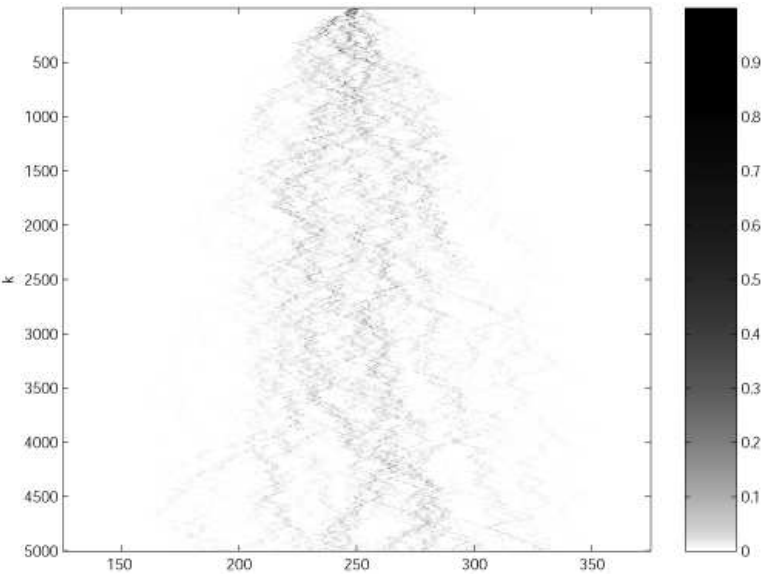

Figure 2: a sample of the measure-valued process $\left\{\nu_{k}^{(100)}, 0 \leq k \leq 5000\right\}$

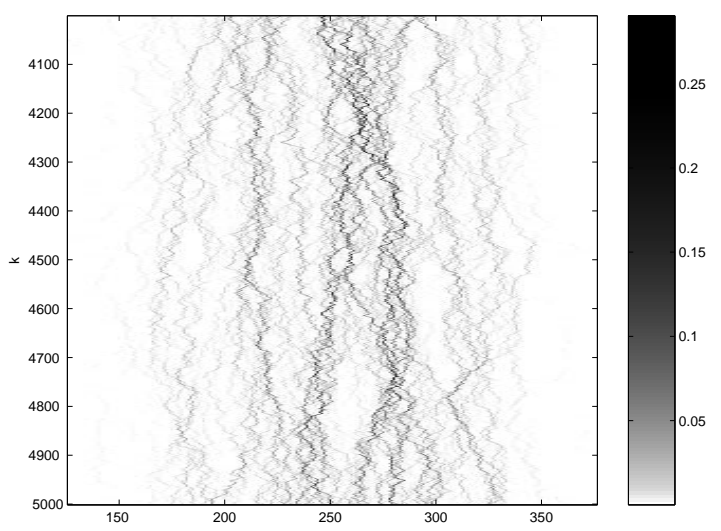

Figure 4: an enlargement of the bottom of figure 2
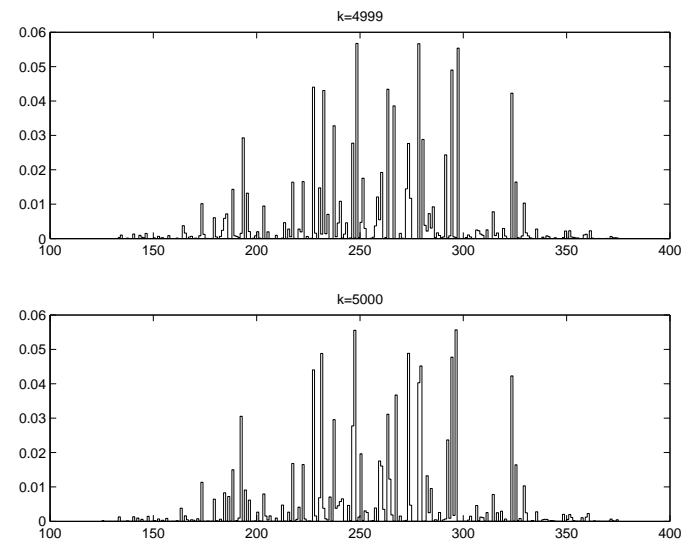

Figure 6: histograms of the sample of the probability measure $\nu_{k}^{(100)}$ for two successive values of $k: k=4999$ and $k=5000$ 


\subsection{Consistent system of Brownian sticky kernels on $S^{1}$}

In [5, 4, a general class of sticky kernels associated with systems of coalescing particles that move as independent Levy processes is constructed. Sticky kernels are characterized by a parameter of stickiness $\tau$ and the exponent $\psi$ of the associated Levy process, that is $\psi(u)=\frac{u^{2}}{2}$ in the case of Brownian sticky kernels. Let us describe the properties of Brownian sticky kernels on $S^{1}$.

For $n \in \mathbb{N}^{*}$, let us consider the measure $m^{(n)}$ defined on $\left(S^{1}\right)^{n}$ by $m^{(n)}=\sum_{\pi \in \mathcal{P}_{n}} p_{\pi}^{(a)} \lambda_{\pi}$ where $\lambda_{\pi}$ is the image of the Lebesgue measure $\lambda^{\otimes|\pi|}$ on $\left(S^{1}\right)^{|\pi|}$ by the one-to-one map $\phi_{\pi}$ (that sends $\left(S^{1}\right)^{|\pi|}$ onto $\left.\left(S^{1}\right)^{n}\right)$ and $\left\{p_{\pi}^{(a)}, \pi \in \mathcal{P}_{n}\right\}$ is the partition function defined in proposition 4 . As this defines an exchangeable and consistent system of measures, it follows from Kingman's representation theorem that there exists a random measure on $S^{1}$ such that $m^{(n)}=E\left(\mu^{\otimes n}\right)$ for every $n \in \mathbb{N}^{*}$. In our case, $\mu$ is the Dirichlet process ${ }^{1}$ of parameter $a \lambda$ on $S^{1}$.

Using this family of measures, a consistent system of Feller semigroups denoted by $\left(P_{t}^{(n)}\right)$ can be defined via their Dirichlet forms:

Proposition 6 (Y. Le Jan and O. Raimond, [5]) For $k \in \mathbb{N}^{*}$, let $\mathcal{E}^{\odot k}$ be the Dirichlet form defined on $L^{2}\left(\left(S^{1}\right)^{k}, \lambda^{\otimes k}\right)$, associated with $k$ independent Brownian motions on $S^{1}$. For every $n \in \mathbb{N}^{*}$, let $\mathcal{E}^{(n)}$ denote the Dirichlet form on $C^{1}\left(\left(S^{1}\right)^{n}\right)$ defined as follows:

$$
\forall f, g \in C^{1}\left(\left(S^{1}\right)^{n}\right), \mathcal{E}^{(n)}(f, g)=\sum_{\pi \in \mathcal{P}_{n}} p_{\pi}^{(a)} \mathcal{E}^{\odot|\pi|}\left(f \circ \phi_{\pi}, g \circ \phi_{\pi}\right) .
$$

- The semigroups $\left(P_{t}^{(n)}\right), n \in \mathbb{N}^{*}$ associated with the Dirichlet forms $\mathcal{E}^{(n)}, n \in \mathbb{N}^{*}$ define a consistent system of strong Feller semigroups.

- For every $n \in \mathbb{N}^{*}$, the generator of $\mathcal{E}^{(n)}$ denoted by $A^{(n)}$ has the following expression on $C^{2}$ functions:

$$
A^{(n)}(f)=\frac{1}{2} \sum_{\pi \in \mathcal{P}_{n}}\left(\Delta^{(|\pi|)}\left(f \circ \phi_{\pi}\right)\right) \circ \phi_{\pi}^{-1} \mathbf{1}_{C_{\pi}} \quad \forall f \in C^{2}\left(\left(S^{1}\right)^{n}\right)
$$

Since $\left\{\left(P_{t}^{(n)}\right)_{t}, n \in \mathbb{N}^{*}\right\}$ is a compatible family of Feller semigroups on $S^{1}$, it follows from theorem 1.1.4 in [3] that it is possible to construct a stochastic flow of kernels $\left(K_{s, t}\right)$ such that $E\left(K_{0, t}^{\otimes n}\right)=P_{t}^{(n)}$ for every $t \in \mathbb{R}_{+}$and $n \in \mathbb{N}^{*}$. This stochastic flow was named sticky flow of parameter $\tau=\frac{1}{a+1}$ and exponent $\psi(u)=\frac{u^{2}}{2}$ (or Brownian sticky flow of parameter $\left.\tau=\frac{1}{a+1}\right)$.

Given a probability measure $\nu_{0}$ on $S^{1}$, the sticky flow induces a stochastic process $\left(\nu_{0} K_{0, t}\right)_{t}$ on the set $\mathcal{M}_{1}\left(S^{1}\right)$ of probability measures on $S^{1}$.

Proposition 7 (Y. Le Jan and O. Raimond, [5, 4]) Let $\left(K_{s, t}\right)_{s \leq t}$ be a sticky Brownian flow of parameter $\tau=\frac{1}{a+1}$.

\footnotetext{
${ }^{1}$ Let $\delta$ be a non null finite measure on a measurable space $(Y, \mathcal{Y})$. A Dirichlet process on $(Y, \mathcal{Y})$ of parameter $\delta$ is a random measure $\mu$ on $Y$ such that for any finite measurable partition $\left(B_{1}, \ldots, B_{r}\right)$ of $Y,\left(\mu\left(B_{1}\right), \ldots, \mu\left(B_{r}\right)\right)$ has the Dirichlet law $\mathcal{D}\left(\delta\left(B_{1}\right), \ldots, \delta\left(B_{r}\right)\right)$.
} 
1. Let $\nu_{0}$ be a probability measure on $S^{1}$. Then $\left(\nu_{0} K_{0, t}\right)_{t}$ is a Feller process on $\mathcal{M}_{1}\left(S^{1}\right)$. It converges in law to the Dirichlet process of parameter a $\lambda$ on $S^{1}$.

2. Let $\mu$ be a Dirichlet process of parameter a $\lambda$ on $S^{1}$, independent of $\left(K_{0, t}\right)_{t}$. Then $\left(\mu K_{0, t}\right)_{t}$ is a reversible process.

\section{Convergence theorem}

We shall now establish the "weak" convergence of the Beta flow of parameter $a$ on $T_{N}$ to the Brownian sticky flow of parameter $\tau=\frac{1}{1+a}$. We keep the same notations as those introduced in section 3 .

Theorem 8 Let $\ell$ be a positive integer. For $N \in \mathbb{N}^{*}$, let $\nu_{N}$ be a probability measure on $\left(\frac{1}{N}(\mathbb{Z} / N \mathbb{Z})\right)^{\ell}$. Assume that $\nu_{N}$ converges weakly to a probability measure $\nu$ on $\left(S^{1}\right)^{\ell}$ as $N$ tends to $+\infty$.

(i) For every $t \in \mathbb{R}_{+}$, the sequence of measure-valued random variables $\nu_{N} K_{N, 0, t}^{\otimes \ell}$ converges in law to $\nu K_{0, t}^{\otimes \ell}$ as $N$ tends to $+\infty$.

(ii) For every continuous functions $f$ and $g$ on $\left(S^{1}\right)^{\ell}$ and for every $t \in \mathbb{R}_{+}$,

$$
\int P_{N, 0, t}^{(\ell)}(g)(x) f(x) d \nu_{N}(x) \text { converges to } \int P_{0, t}^{(\ell)}(g)(x) f(x) d \nu(x)
$$

as $N$ tends to $+\infty$.

For $N \in \mathbb{N}^{*}$, let $\eta_{N}$ be a probability measure on $\frac{1}{N}(\mathbb{Z} / N \mathbb{Z})$. Assume that $\left(\eta_{N}\right)_{N}$ converges weakly to a probability measure $\eta$ on $S^{1}$.

(iii) The finite dimensional distributions of the measure-valued Markov process $\left\{\eta_{N} K_{N, 0, t}, t \in \mathbb{R}_{+}\right\}$weakly converge to the finite dimensional distributions of $\left\{\eta K_{0, t}, t \in \mathbb{R}_{+}\right\}$as $N$ tends to $+\infty$.

\section{Remark.}

1. Assertions of the theorem also hold with sequences of probability measures $\nu_{N}$ and $\eta_{N}$ defined on $\left(\frac{1}{2 N}((2 \mathbb{Z}+1) / 2 N \mathbb{Z})\right)^{\ell}$ and $\frac{1}{2 N}((2 \mathbb{Z}+1) / 2 N \mathbb{Z})$ respectively that converge weakly as $N$ tends to $+\infty$.

2. For $N \in \mathbb{N}^{*}$, let $\nu_{N}^{(1)}, \ldots, \nu_{N}^{(r)}$ be probability measures on $\frac{1}{N}(\mathbb{Z} / N \mathbb{Z})$ such that $\left(\nu_{N}^{(i)}\right)_{N}$ converges weakly to a probability measure $\nu^{(i)}$ of $S^{1}$ for every $i \in\{1, \ldots, r\}$. It follows from assertion $(i)$ of theorem 8 that $\left(\nu_{N}^{(1)} K_{N, 0, t}, \ldots, \nu_{N}^{(r)} K_{N, 0, t}\right)$ converges in law to $\left(\nu^{(1)} K_{0, t}, \ldots, \nu^{(r)} K_{0, t}\right)$ for every $t \in \mathbb{R}_{+}$. This property could be used as a definition of the convergence in law of stochastic flows of kernels with independent increments.

Before going into details, let us explain the scheme of the proof of theorem 8 . First, (ii) and (iii) will be deduced from assertion $(i)$ of the theorem. The main step of the proof of $(i)$ is to show the following convergence of the resolvents: 
Proposition 9 Let $n \in \mathbb{N}^{*}$. Let $\left(V_{N, \alpha}^{(n)}\right)_{\alpha>0}$ denote the resolvent associated with the n-point motion of the Beta flow of parameter a on $T_{N}^{(n)}$ and let $\left(V_{\alpha}^{(n)}\right)_{\alpha>0}$ denote the resolvent of the $n$-point motion of the Brownian sticky flow of parameter $\tau=\frac{1}{a+1}$. For every $\alpha \in \mathbb{R}_{+}^{*}$ and continuous functions $f$ and $g$ on $\left(S^{1}\right)^{n}, \int V_{N, \alpha}^{(n)}(f) g d m_{N}^{(n)}$ converges to $\int V_{\alpha}^{(n)}(f) g d m^{(n)}$ as $N$ tends to $+\infty$.

As the discrete and the continuous $n$-point processes are both reversible, an argument using spectral measures allows to deduce the following weak convergence of the semigroups from the weak convergence of the resolvents:

Proposition 10 Let $n \in \mathbb{N}^{*}$. If $f$ and $g$ are continuous functions on $\left(S^{1}\right)^{n}$, then $\int g P_{N, t}^{(n)}(f) d m_{N}^{(n)}$ converges to $\int g P_{t}^{(n)}(f) d m^{(n)}$ as $N$ tends to $+\infty$.

A last step consists in making the previous convergence result of the semigroups also true if $m_{N}^{(n)}$ is replaced by any sequence of probability measures $\nu_{N}$ on $\left(\frac{1}{N}(\mathbb{Z} / N \mathbb{Z})\right)^{n}$ that weakly converges.

\subsection{Convergence of the resolvents of the $n$-point motions}

The convergence of the resolvents is based on the convergence of the invariant measures $m_{N}^{(n)}$ and of the generator of the $n$-point motion of the flow of Beta matrices together with a Lipschitz property of the discrete resolvent $V_{N, \alpha}^{(n)}$. Before proving proposition 9, let us give precise statements of these three points.

\subsubsection{Convergence of the invariant measures}

Lemma 11 For every partition $\pi$ of $[n]$, let $f_{\pi}$ be a function defined on $\left(S^{1}\right)^{n}$, Lipschitz on $E_{\pi}$ and vanishing outside $E_{\pi}$. Let $f=\sum_{\pi \in \mathcal{P}_{n}} f_{\pi}$. Then

$$
\left|\int f d m_{N}^{(n)}-\int f d m^{(n)}\right| \leq \frac{C_{n}}{N} \sum_{\pi \in \mathcal{P}_{n}}\left(\left\|f_{\pi}\right\|_{L i p}+\left\|f_{\pi}\right\|_{\infty}\right) .
$$

Proof. Let $E_{N, \pi}$ denote the intersection of $E_{\pi}$ with $T_{N}^{(n)}$ and let $\lambda_{N, \pi}$ be the uniform distribution on $E_{N, \pi}$. By formula (4) and proposition 4 $m_{N}^{(n)}=\sum_{\pi \in \mathcal{P}_{n}} p_{\pi}^{(a)} \lambda_{N, \pi}$. As the measure $m^{(n)}$ has a similar decomposition: $m^{(n)}=\sum_{\pi \in \mathcal{P}_{n}} p_{\pi}^{(a)} \lambda_{\pi}$, it suffices to prove that for all partitions $\pi, \pi^{\prime}$ of $[n]$,

$$
\left|\int_{E_{N, \pi^{\prime}}} f_{\pi} d \lambda_{N, \pi^{\prime}}-\int_{E_{\pi^{\prime}}} f_{\pi} d \lambda_{\pi^{\prime}}\right| \leq \frac{C_{n}}{N}\left(\left\|f_{\pi}\right\|_{L i p}+\left\|f_{\pi}\right\|_{\infty}\right) .
$$

Let us first consider the case $\pi$ is not finer than $\pi^{\prime}$, that is there is a nonempty block $B$ of $\pi$ intersecting at least two blocks of $\pi^{\prime}$. Let $\tilde{\pi}$ be the partition of $[n]$ obtained by merging the blocks of $\pi$ that intersect the same block of $\pi^{\prime}$. Then $E_{\pi^{\prime}} \cap E_{\pi}$ is a subset of $E_{\tilde{\pi}}$. As $\tilde{\pi}$ is a coarser partition than $\pi^{\prime}, \lambda_{\pi^{\prime}}\left(E_{\tilde{\pi}}\right)=0$ and $\left|E_{N, \tilde{\pi}}\right| \leq \frac{1}{N}\left|E_{N, \pi^{\prime}}\right|$. Thus

$$
\left|\int_{E_{N, \pi^{\prime}}} f_{\pi} d \lambda_{N, \pi^{\prime}}-\int_{E_{\pi^{\prime}}} f_{\pi} d \lambda_{\pi^{\prime}}\right|=\frac{1}{\left|E_{N, \pi^{\prime}}\right|}\left|\sum_{x \in E_{N, \pi^{\prime}} \cap E_{N, \pi}} f_{\pi}(x)\right| \leq \frac{1}{N}\left\|f_{\pi}\right\|_{\infty} .
$$


Let us now consider the case $\pi$ is equal to $\pi^{\prime}$ or finer than $\pi^{\prime}$. Then $E_{\pi^{\prime}} \subset E_{\pi}$. If $\pi^{\prime}$ has $k$ nonempty blocks then

$$
\left|\int_{E_{N, \pi^{\prime}}} f_{\pi} d \lambda_{N, \pi^{\prime}}-\int_{E_{\pi^{\prime}}} f_{\pi} d \lambda_{\pi^{\prime}}\right|=\left|\frac{1}{2 N^{k}} \sum_{x \in T_{N}^{(k)}} f_{\pi}\left(\phi_{\pi^{\prime}}(x)\right)-\int_{\left(S^{1}\right)^{k}} f_{\pi^{\prime}}\left(\phi_{\pi^{\prime}}(x)\right) d x\right| .
$$

The function $f_{\pi^{\prime}} \circ \phi_{\pi^{\prime}}$ is a Lipschitz function with Lipschitz coefficient smaller than $n\left\|f_{\pi^{\prime}}\right\|_{\text {Lip }}$. Thus it remains to establish the following result: for every $k \in \mathbb{N}^{*}$, there exists a constant $C_{k}$ such that for every Lipschitz function $g$ on $\left(S^{1}\right)^{k}$,

$$
\left|\frac{1}{2 N^{k}} \sum_{x \in T_{N}^{(k)}} g(x)-\int_{\left(S^{1}\right)^{k}} g(x) d x\right| \leq \frac{C_{k}}{N}\|g\|_{\text {Lip }} .
$$

The proof can be made by induction on $k$.

\subsubsection{Convergence of the generators}

Lemma 12 For every $n \in \mathbb{N}^{*}$, let $A_{N}^{(n)}$ denote the generator of the $n$-point motion of the Beta flow on $T_{N}^{(n)}$ of parameter a. For every $C^{2}$ function $f$ on $\left(S^{1}\right)^{n}$,

$$
\sup _{x \in T_{N}^{(n)}}\left|A_{N}^{(n)}(f)(x)-A^{(n)}(f)(x)\right|
$$

converges to 0 as $N$ tends to $+\infty$.

Proof. Let $n \in \mathbb{N}^{*}$ and $f$ be a $C^{2}$ function defined on $\left(S^{1}\right)^{n}$. Let us recall the expression of $A^{(n)}(f)$ :

$$
A^{(n)}(f)=\frac{1}{2} \sum_{\pi \in \mathcal{P}_{n}} \Delta_{\pi}(f) \mathbf{1}_{C_{\pi}} \text { where } \Delta_{\pi}(f)(x)=\Delta^{(|\pi|)}\left(f \circ \phi_{\pi}\right)\left(\phi_{\pi}^{-1}(x)\right) .
$$

Thus it suffices to prove that for every partition $\pi$ of $[n]$,

$$
\sup _{x \in C_{\pi} \cap T_{N}^{(n)}}\left|2 A_{N}^{(n)}(f)(x)-\Delta_{\pi} f(x)\right|
$$

converges to 0 as $N$ tends to $+\infty$.

We shall introduce the discrete version of the Laplacian $\Delta_{\pi}$, denoted by $\Delta_{N, \pi}$ and we shall prove that the difference between $2 A_{N}^{(n)}(f)$ and $\Delta_{N, \pi} f$ goes to zero as $N$ tends to $+\infty$.

The expression linking the generator $A_{N}^{(n)}$ and the transition matrix $P_{N}^{(n)}$ is the following: for a function $g$ defined on $\left(S^{1}\right)^{n}$ and $x \in T_{N}^{(n)}$,

$$
A_{N}^{(n)}(g)(x)=4 N^{2}\left(\sum_{\varepsilon \in\left\{ \pm \frac{1}{2 N}\right\}^{n}} P_{N}^{(n)}(x, x+\varepsilon) g(x+\varepsilon)-g(x)\right) .
$$

Let us recall the expression of $P_{N}^{(n)}$ : for every $x \in T_{N}^{(n)}$ and $\varepsilon \in\left\{ \pm \frac{1}{2 N}\right\}^{n}$,

$$
P_{N}^{(n)}(x, x+\varepsilon)=\prod_{l \in \mathbb{Z} / 2 N \mathbb{Z}} \frac{\prod_{i=0}^{s_{l}^{+}(x, \varepsilon)-1}\left(\frac{a}{2 N}+i\right) \prod_{i=0}^{s_{l}^{-}(x, \varepsilon)-1}\left(\frac{a}{2 N}+i\right)}{\prod_{i=0}^{s_{l}(x)-1}\left(\frac{a}{N}+i\right)} .
$$


where $s_{l}(x)$ denotes the number of coordinates of $2 N x$ equal to $l$ and

$$
s_{l}^{ \pm}(x, \varepsilon)=\operatorname{Card}\left(\left\{i \in\{1, \ldots, n\}, 2 N x_{i}=l \text { and } 2 N \varepsilon_{i}= \pm 1\right\}\right) .
$$

As $s_{l}^{+}(x, \varepsilon)=s_{l}^{-}(x,-\varepsilon), P_{N}^{(n)}(x, x+\varepsilon)=P_{N}^{(n)}(x, x-\varepsilon)$. Thus,

$$
2 A_{N}^{(n)}(g)(x)=\sum_{\varepsilon \in\left\{ \pm \frac{1}{2 N}\right\}^{n}} P_{N}^{(n)}(x, x+\varepsilon) L_{n}(g)(x, \varepsilon) \forall x \in T_{N}^{(n)},
$$

where $L_{n}(g)(x, h)=4 N^{2}(g(x+h)+g(x-h)-2 g(x))$ for $h \in \mathbb{R}$.

For $d, N \in \mathbb{N}^{*}$, let $\Delta_{N}^{(d)}$ denote the discrete Laplacian on the set $T_{N}^{(d)}$. Its expression in terms of $L_{d}$ is:

$$
\Delta_{N}^{(d)} g=\frac{1}{2^{d}} \sum_{\epsilon \in\{ \pm 1\}^{d}} L_{d}(g)\left(\cdot, \frac{\epsilon}{2 N}\right)
$$

for a function $g$ defined on $T_{N}^{(d)}$. Let $\pi$ be a partition of $[n]$ having $d$ nonempty blocks and set $\Delta_{N, \pi} g(\cdot)=\Delta_{N}^{(d)}\left(g \circ \phi_{\pi}\right)\left(\phi_{\pi}^{-1}(\cdot)\right)$ for a function $g$ defined on $T_{N}^{(n)}$. As the restriction of $\phi_{\pi}$ to $\{ \pm 1\}^{d}$ is a one-to-one map onto $E_{\pi} \cap\{ \pm 1\}^{n}$,

$$
\Delta_{N, \pi} f(x)=\frac{1}{2^{d}} \sum_{\varepsilon \in\left\{ \pm \frac{1}{2 N}\right\}^{n} \cap E_{\pi}} L_{n}(f)(x, \varepsilon) .
$$

Consequently, the expression of $2 A_{N}^{(n)} f(x)-\Delta_{\pi} f(x)$ can be split into the three following terms:

$$
\begin{aligned}
I_{N}^{1}(x) & =\sum_{\varepsilon \in E_{\pi} \cap\left\{ \pm \frac{1}{2 N}\right\}^{n}}\left(P_{N}^{(n)}(x, x+\varepsilon)-\frac{1}{2^{d}}\right) L_{n}(f)(x, \varepsilon) \\
I_{N}^{2}(x) & =\Delta_{N, \pi} f(x)-\Delta_{\pi} f(x) \\
I_{N}^{3}(x) & =\sum_{\varepsilon \in E_{\pi}^{c \cap\left\{ \pm \frac{1}{2 N}\right\}^{n}}} P_{N}^{(n)}(x, x+\varepsilon) L_{n}(f)(x, \varepsilon)
\end{aligned}
$$

Asymptotic behaviour of $I_{N}^{2}(x)$. For a $C^{2}$ function $g$ on $\left(S^{1}\right)^{r}, x \in\left(S^{1}\right)^{r}$ and $\epsilon \in\{ \pm 1\}^{r}$, set $\mathcal{L}_{r}(g)(x, \epsilon)=\sum_{i=1}^{r} \sum_{j=1}^{r} \epsilon_{i} \epsilon_{j} \partial_{i, j}^{2} g(x)$. It follows from a Taylor expansion with integral remainder that $\left|L_{r}(g)\left(x, \frac{\epsilon}{2 N}\right)-\mathcal{L}_{r}(g)(x, \epsilon)\right|$ converges to zero uniformly on $x \in T_{N}^{(r)}$ and $\epsilon \in\{ \pm 1\}^{r}$ as $N$ tends to $+\infty$. Thus $I_{N}^{2}(x)=\Delta_{N, \pi} f(x)-\Delta_{\pi} f(x)$ converges to zero uniformly on $x \in T_{N}^{(n)}$.

Asymptotic behaviour of $P_{N}^{(n)}(x, x+\varepsilon)$. Let $\left(B_{1}, \ldots, B_{d}\right)$ denote the nonempty blocks of $\pi$ and let $x \in C_{\pi} \cap T_{N}^{(n)}$. Then

$$
P_{N}^{(n)}(x, x+\varepsilon)=\prod_{l=1}^{d} \frac{\gamma_{\frac{a}{2 N}}\left(\sum_{i \in B_{l}} \mathbf{1}_{\left\{\varepsilon_{i}=\frac{1}{2 N}\right\}}\right) \gamma_{\frac{a}{2 N}}\left(\sum_{i \in B_{l}} \mathbf{1}_{\left\{\varepsilon_{i}=-\frac{1}{2 N}\right\}}\right)}{\gamma_{\frac{a}{N}}\left(\left|B_{l}\right|\right)} \forall \varepsilon \in\left\{ \pm \frac{1}{2 N}\right\}^{n}
$$

where $\gamma_{b}(u)$ denotes the product $\prod_{i=0}^{u-1}(b+i)$ for $b>0$ and $u \in \mathbb{N}$ with the convention $\prod_{i=0}^{-1}=1$. A computation shows that 
- if $u, v \in \mathbb{N}^{*}$ then

$$
\frac{\gamma_{\frac{a}{2 N}}(u) \gamma_{\frac{a}{2 N}}(v)}{\gamma_{\frac{a}{N}}(u+v)} \leq \frac{a}{4 N} \frac{u ! v !}{(u+v-1) !} \text { for } 2 N \geq a .
$$

- if $u \in \mathbb{N}^{*}$ and $v=0$ then

$$
\begin{aligned}
\left|\frac{\gamma_{\frac{a}{2 N}}(u) \gamma_{\frac{a}{2 N}}(0)}{\gamma_{\frac{a}{N}}(u)}-\frac{1}{2}\right| & =\frac{1}{2}\left(1-\prod_{i=1}^{u-1}\left(1-\frac{a}{2 a+2 N i}\right)\right) \\
& \leq \frac{1}{2}\left(1-\left(1-\frac{a}{2 a+2 N}\right)^{u-1}\right) .
\end{aligned}
$$

Thus

$$
\sup _{x \in C_{\pi}, \varepsilon \in\left\{ \pm \frac{1}{2 N}\right\}^{n} \cap E_{\pi}^{c}} P_{N}^{(n)}(x, x+\varepsilon) \text { and } \sup _{x \in C_{\pi}, \varepsilon \in\left\{ \pm \frac{1}{2 N}\right\}^{n} \cap E_{\pi}}\left|P_{N}^{(n)}(x, x+\varepsilon)-\frac{1}{2^{d}}\right|
$$

converge to 0 as $N$ tends to $+\infty$.

\subsubsection{Lipschitz property of the resolvents and the semigroups associated with the Beta flow}

Lemma 13 Let $\left(P_{N, t}^{(n)}\right)_{t}$ denote the n-point Markovian semigroup of the Beta flow on $T_{N}^{(n)}$. If $f:\left(S^{1}\right)^{n} \rightarrow \mathbb{R}$ is a Lipschitz function then

- the Lipschitz coefficient of $P_{N, t}^{(n)}(f)$ is bounded by $\|f\|_{\text {Lip }}$.

- the Lipschitz coefficient of $V_{N, \alpha}^{(n)}(f)$ is bounded by $\frac{1}{\alpha}\|f\|_{L i p}$.

Proof. We use a coupling argument borrowed from $\underline{5}$. Let $\underline{x}=\left(x_{1}, \ldots, x_{n+1}\right)$ be a point of $T_{N}^{(n+1)}$ such that $x_{1} \neq x_{2}$ and let $X_{t}=\left(X_{t}^{(1)}, \ldots, X_{t}^{(n+1)}\right)$ be a Markov chain on $T_{N}^{(n+1)}$ with transition matrix $P_{N}^{(n+1)}$ and with initial point $\underline{x}$. Set $\tau=\inf \left\{s>0, X_{s}^{(1)}=X_{s}^{(2)}\right\}$. Since $\left(X_{t}^{(1)}, X_{t}^{(2)}\right)$ is a positive recurrent Markov chain on $T_{N}^{(2)}, \tau$ is almost surely finite. Let us define two processes $\left(Y_{t}\right)_{t}$ and $\left(Z_{t}\right)_{t}$ on $T_{N}^{(n)}$ :

- $Y_{t}^{(1)}=X_{t}^{(1)}$ and $Y_{t}^{(i)}=X_{t}^{(i+1)}$ for $i \in\{2, \ldots, n\}$,

- $Z_{t}^{(1)}=X_{t}^{(2)} \mathbf{1}_{t \leq \tau}+X_{t}^{(1)} \mathbf{1}_{t>\tau}$ and $Z_{t}^{(i)}=X_{t}^{(i+1)}$ for $i \in\{2, \ldots, n\}$.

As $\left\{\left(P_{N, t}^{(n)}\right)_{t}, n \in \mathbb{N}^{*}\right\}$ defines a consistent family of Markovian semigroups, $\left(Y_{t}\right)_{t}$ and $\left(X_{t}^{(2)}, X_{t}^{(3)}, \ldots, X_{t}^{(n+1)}\right)_{t}$ are both Markov processes with semigroup $\left(P_{N, t}^{(n)}\right)_{t}$. The strong Markov property implies that $\left(Z_{t}\right)_{t}$ is also a Markov process with semigroup $\left(P_{N, t}^{(n)}\right)_{t}$. As $Y_{t}=Z_{t}$ if $t \geq \tau$

$$
\begin{aligned}
\mid P_{N, t}^{(n)}(f)\left(x_{1}, x_{3}, \ldots, x_{n+1}\right)-P_{N, t}^{(n)}(f) & \left(x_{2}, x_{3}, \ldots, x_{n+1}\right) \mid \\
& =\left|E\left(f\left(Y_{t \wedge \tau}\right)-f\left(Z_{t \wedge \tau}\right)\right)\right| \leq\|f\|_{L i p} E\left(d\left(X_{t \wedge \tau}^{(1)}, X_{t \wedge \tau}^{(2)}\right)\right) .
\end{aligned}
$$


and

$$
\begin{aligned}
\mid V_{N, \alpha}^{(n)}(f)\left(x_{1}, x_{3}, \ldots, x_{n+1}\right) & -V_{N, \alpha}^{(n)}(f)\left(x_{2}, x_{3}, \ldots, x_{n+1}\right) \mid \\
=\mid \int_{0}^{+\infty} E\left(f\left(Y_{t \wedge \tau}\right)-\right. & \left.f\left(Z_{t \wedge \tau}\right)\right) e^{-\alpha t} d t \mid \\
& \leq\|f\|_{\text {Lip }} \int_{0}^{+\infty} e^{-\alpha t} E\left(d\left(X_{t \wedge \tau}^{(1)}, X_{t \wedge \tau}^{(2)}\right)\right) d t .
\end{aligned}
$$

Let us show that for every $t \geq 0, E\left(d\left(X_{t \wedge \tau}^{(1)}, X_{t \wedge \tau}^{(2)}\right)\right) \leq d\left(x_{1}, x_{2}\right)$. Without loss of generality, one may assume that $w=x_{1}-x_{2} \in\left\{0, \frac{1}{N}, \ldots, \frac{N-1}{N}\right\}$. Let $\left(\hat{X}_{t}^{(1)}, \hat{X}_{t}^{(2)}\right)$ be a Markov chain on $\left(\frac{1}{2 N} \mathbb{Z}\right)^{2}$ starting from $\left(x_{1}, x_{2}\right)$ whose transition matrix $\hat{P}$ is defined by:

$$
\hat{P}(x+k, y+l)=P_{N}^{(2)}(x, y) \text { for every }(x, y) \in T_{N}^{(2)} \text { and } k, l \in \mathbb{Z}^{2} .
$$

Set $W_{t}=\hat{X}_{t}^{(1)}-\hat{X}_{t}^{(2)}$. Since $2 N w$ is even, for every $t \geq 0, W_{t \wedge \tau}$ remains nonnegative, whence $E\left(d\left(\hat{X}_{t \wedge \tau}^{(1)}, \hat{X}_{t \wedge \tau}^{(2)}\right)\right) \leq E\left(W_{t \wedge \tau}\right)$. As $\left(W_{t}\right)$ is a martingale, for every $t \geq 0$, $E\left(W_{t \wedge \tau}\right)=w$. Therefore, for every $t \geq 0$,

$$
E\left(d\left(X_{t \wedge \tau}^{(1)}, X_{t \wedge \tau}^{(2)}\right)\right)=E\left(d\left(\hat{X}_{t \wedge \tau}^{(1)}, \hat{X}_{t \wedge \tau}^{(2)}\right)\right) \leq w=d\left(x_{1}, x_{2}\right) .
$$

We have obtained the following inequalities: for every $t \geq 0$,

$$
\begin{aligned}
& \left|P_{N, t}^{(n)}(f)\left(x_{1}, x_{3}, \ldots, x_{n+1}\right)-P_{N, t}^{(n)}(f)\left(x_{2}, x_{3}, \ldots, x_{n+1}\right)\right| \leq\|f\|_{L i p} d\left(x_{1}, x_{2}\right), \\
& \left|V_{N, \alpha}^{(n)}(f)\left(x_{1}, x_{3}, \ldots, x_{n+1}\right)-V_{N, \alpha}^{(n)}(f)\left(x_{2}, x_{3}, \ldots, x_{n+1}\right)\right| \leq \frac{1}{\alpha}\|f\|_{L i p} d\left(x_{1}, x_{2}\right) .
\end{aligned}
$$

As the semigroup $\left(P_{N, t}^{(n)}\right)_{t}$ is invariant by the action of a permutation, for every $x, y \in T_{N}^{(n)}$,

$$
\begin{aligned}
& \left|P_{N, t}^{(n)}(f)(x)-P_{N, t}^{(n)}(f)(y)\right| \leq\|f\|_{\text {Lip }} \sum_{i=1}^{n} d\left(x_{i}, y_{i}\right), \\
& \left|V_{N, \alpha}^{(n)}(f)(x)-V_{N, \alpha}^{(n)}(f)(y)\right| \leq \frac{1}{\alpha}\|f\|_{\text {Lip }} \sum_{i=1}^{n} d\left(x_{i}, y_{i}\right) .
\end{aligned}
$$

\subsubsection{Proof of proposition 9 ,}

A density argument reduces the problem to showing that for all $C^{1}$ functions $f$ and $g$ on $\left(S^{1}\right)^{n}, \int V_{N, \alpha}^{(n)}(f) g d m_{N}^{(n)}$ converges to $\int V_{\alpha}^{(n)}(f) g d m^{(n)}$ as $N$ tends to $+\infty$.

Let us introduce an extension of $V_{N, \alpha}^{(n)}(f)$ to $\left(S^{1}\right)^{n}$ :

Lemma 14 A function $g$ on $T_{N}^{(n)}$ can be extended to a Lipschitz function $\tilde{g}$ on $\left(S^{1}\right)^{n}$ such that:

- $\|\tilde{g}\|_{\infty}=\|g\|_{\infty}$

- $\|\tilde{g}\|_{\text {Lip }} \leq C_{n}\|g\|_{\text {Lip }}$ where $C_{n}$ is a constant only depending on $n$.

- $\tilde{g}$ is differentiable on $\left(S^{1}\right)^{n}-\mathcal{R}$ where $\mathcal{R}$ is the subset of points having at least one coordinate in $\frac{1}{2 N}(\mathbb{Z} / 2 N \mathbb{Z})$ and $\left\|\partial_{i} \tilde{g}(x)\right\| \leq C_{n}\|g\|_{\text {Lip }}$ for all $i \in\{1, \ldots, n\}$ and $x \in\left(S^{1}\right)^{n}-\mathcal{R}$. 
Proof. Firstly, a function $g$ on $T_{N}^{(n)}$ is extended to a function $\bar{g}$ on the lattice $\left(\frac{1}{2 N}(\mathbb{Z} / 2 N \mathbb{Z})\right)^{n}$ as follows: for $x \in\left(\frac{1}{2 N}(\mathbb{Z} / 2 N \mathbb{Z})\right)^{n}-T_{N}^{(n)}$, set

$$
\bar{g}(x)=\frac{1}{\left|V_{x}\right|} \sum_{y \in V_{x}} g(y)
$$

where $V_{x}$ is the set of the nearest points of $x$ in $\left(\frac{1}{2 N}(\mathbb{Z} / 2 N \mathbb{Z})\right)^{n}$ in the sense of the distance $d_{n}(x, y)=\sum_{i=1}^{n} d\left(x_{i}, y_{i}\right)$. This extension has the following properties:

- $\|\bar{g}\|_{\infty}=\|g\|_{\infty}$,

- there is a constant $C_{n}>0$ such that for every function $g: T_{N}^{(n)} \rightarrow \mathbb{R},\|\bar{g}\|_{\text {Lip }} \leq C_{n}\|g\|_{\text {Lip }}$.

Lastly, a function $f$ defined on $\left(\frac{1}{2 N}(\mathbb{Z} / 2 N \mathbb{Z})\right)^{n}$ is extended to a function $\hat{f}$ on $\left(S^{1}\right)^{n}$ as follows. A point $x=\left(x_{1}, \ldots, x_{n}\right)$ in an elementary cube $\left.\prod_{i=1}^{n}\right] \frac{k_{i}}{2 N}, \frac{k_{i}+1}{2 N}[$ is the barycentre of the vertices of this cube $\frac{k+\eta}{2 N}, \eta \in\{0,1\}^{n}$ with the weights

$$
\alpha_{n}(k+\eta, x)=(2 N)^{n} \prod_{i=1}^{n}\left(x_{i}-\frac{k_{i}}{2 N}\right)^{\eta_{i}}\left(\frac{k_{i}+1}{2 N}-x_{i}\right)^{1-\eta_{i}}
$$

respectively. Then we set $\hat{f}(x)$ as the convex combination of the points $f\left(\frac{k+\eta}{2 N}\right)$ with the weights $\alpha_{n}(k+\eta, x)$ for every $\eta \in\{0,1\}^{n}$ :

$$
\hat{f}(x)=\sum_{\eta \in\{0,1\}^{n}} \alpha_{n}(k+\eta, x) f\left(\frac{k+\eta}{2 N}\right) .
$$

Let us list some properties of this extension:

- $\|\hat{f}\|_{\infty}=\|f\|_{\infty}$ and $f$ is differentiable in $\left(S^{1}\right)^{n}-\mathcal{R}$.

- $\|\hat{f}\|_{\text {Lip }} \leq\|f\|_{\text {Lip }}$ and for every $i \in\{1, \ldots, n\},\left|\partial_{i} \hat{f}(x)\right| \leq\|f\|_{\text {Lip }}$ if $x \in\left(S^{1}\right)^{n}-\mathcal{R}$.

By lemma 13, the Lipschitz coefficient of $V_{N, \alpha}^{(n)}(f)$ is bounded by a constant irrespective of $N$, hence it is also the case for $\widehat{V_{N, \alpha}^{(n)}(f)}$. Thus on applying lemma 11, we obtain that the difference between $\int V_{N, \alpha}^{(n)}(f) g d m_{N}^{(n)}$ and $\int \widehat{V_{N, \alpha}^{(n)}(f)} g d m^{(n)}$ converges to zero as $N$ tends to $+\infty$.

The remainder of the proof is the subject of the following key lemma:

Lemma 15 Let $\alpha>0$. If $f$ is a $C^{1}$ function on $\left(S^{1}\right)^{n}$ then for every $g \in L^{2}\left(m^{(n)}\right)$, $\int g \widehat{V_{N, \alpha}^{(n)}(f)} d m^{(n)}$ converges to $\int g V_{\alpha}^{(n)}(f) d m^{(n)}$.

Proof. For a positive real $\alpha$, set $\mathcal{E}_{\alpha}^{(n)}(\cdot, \cdot)=\alpha\langle\cdot, \cdot\rangle_{m^{(n)}}+\mathcal{E}^{(n)}(\cdot, \cdot)$ and $\mathcal{H}^{(n)}$ the closure of $C^{1}\left(\left(S^{1}\right)^{n}\right)$ for the metric $\mathcal{E}_{1}^{(n)}$.

As $\langle u, v\rangle_{m^{(n)}}=\mathcal{E}_{\alpha}^{(n)}\left(V_{\alpha}^{(n)}(u), v\right)$ for every $u \in L^{2}\left(m^{(n)}\right)$ and $v \in \mathcal{H}^{(n)}$, the weak convergence in the Dirichlet space $\left(\mathcal{H}^{(n)}, \mathcal{E}_{\alpha}^{(n)}\right)$ implies the weak convergence in $\left(L^{2}\left(m^{(n)}\right),\langle\cdot, \cdot\rangle_{m^{(n)}}\right)$. 
Thus, it suffices to prove that for all $g \in \mathcal{H}^{(n)}, \mathcal{E}_{\alpha}^{(n)}\left(\widetilde{V_{\alpha, N}^{(n)}(f)}, g\right)$ tends to $\mathcal{E}_{\alpha}^{(n)}\left(V_{\alpha}^{(n)}(f), g\right)$. It follows from lemmas 13 and 14 that $\widetilde{V_{\alpha, N}^{(n)}(f)}$ is bounded by $\frac{\|f\|_{\infty}}{\alpha}$ and differentiable on $\left(S^{1}\right)^{n}-\mathcal{R}$ with partial derivatives bounded by $\|f\|_{\text {Lip }}$. Thus expression (15) of $\mathcal{E}^{(n)}$ enables us to establish the following inequality

$$
\mathcal{E}_{\alpha}^{(n)}\left(\widetilde{V_{\alpha, N}^{(n)}(f)}\right) \leq C_{n, \alpha}\left(\|f\|_{\text {Lip }}^{2}+\|f\|_{\infty}^{2}\right)
$$

where $C_{n, \alpha}$ is a constant irrespective of $N$ and $f$. As the set of $C^{3}$ functions is dense in $\mathcal{H}^{(n)}$, this reduces the problem to proving the convergence of $\mathcal{E}_{\alpha}^{(n)}\left(\widetilde{V_{\alpha, N}^{(n)}(f)}, g\right)$ for any $C^{3}$ function $g$.

Let $g$ be a $C^{3}$ function. The difference $\mathcal{E}_{\alpha}^{(n)}\left(\widetilde{V_{N, \alpha}^{(n)}(f)}, g\right)-\mathcal{E}_{\alpha}^{(n)}\left(V_{\alpha}^{(n)}(f), g\right)$ is the sum of two terms:

$$
\begin{aligned}
I_{N}^{(1)} & =\mathcal{E}_{\alpha}^{(n)}\left(\widetilde{V_{N, \alpha}^{(n)}(f)}, g\right)-\mathcal{E}_{N, \alpha}^{(n)}\left(V_{N, \alpha}^{(n)}(f), g\right) \\
I_{N}^{(2)} & =\int f(x) g(x) d m_{N}^{(n)}(x)-\int f(x) g(x) d m^{(n)}(x)
\end{aligned}
$$

By lemma 11, $I_{N}^{(2)}$ goes to zero as $N$ tends to $+\infty$.

Let us split up $I_{N}^{(1)}$ :

$$
\begin{array}{r}
I_{N}^{(1)}=\int \widehat{V_{N, \alpha}^{(n)}(f)}\left(\alpha-A^{(n)}\right)(g) d m^{(n)}-\int \widehat{V_{N, \alpha}^{(n)}(f)}\left(\alpha-A^{(n)}\right)(g) d m_{N}^{(n)} \\
+\int V_{N, \alpha}^{(n)}(f)\left(A_{N}^{(n)}-A^{(n)}\right)(g) d m_{N}^{(n)}
\end{array}
$$

It follows from expression (6) of the generator $A^{(n)}$ that $A^{(n)}(g)$ is a sum of Lipschitz functions on $E_{\pi}$ that vanish out of $E_{\pi}$. Thus lemma 11 can be applied to $\widetilde{V_{N, \alpha}^{(n)}(f)}\left(\alpha-A^{(n)}\right)$. Finally, the last integral is bounded by

$$
\frac{\|f\|_{\infty}}{\alpha} \sup _{x \in T_{N}^{(n)}}\left|\left(A_{N}^{(n)}-A^{(n)}\right)(g)(x)\right| .
$$

Thus it converges to 0 as $N$ tends to $+\infty$, by lemma 12

\subsection{Proof of proposition 10.}

Let $f$ be a continuous function on $\left(S^{1}\right)^{n}$. As the two $n$-point processes are reversible Markov processes, their generators $A_{N}^{(n)}$ and $A^{(n)}$ are self-adjoint operators on the Hilbert spaces $L^{2}\left(m_{N}^{(n)}\right)$ and $L^{2}\left(m^{(n)}\right)$ with nonpositive spectra. Let $\nu_{N}^{f}$ and $\nu^{f}$ denote the spectral measures of $A_{N}^{(n)}$ and $A^{(n)}$ respectively associated with the function $f$ :

$$
\left\langle f, \psi\left(A_{N}^{(n)}\right) f\right\rangle_{m_{N}^{(n)}}=\int_{\mathbb{R}_{-}} \psi d \nu_{N}^{f} \text { and }\left\langle f, \psi\left(A^{(n)}\right) f\right\rangle_{m^{(n)}}=\int_{\mathbb{R}_{-}} \psi d \nu^{f}
$$


for every continuous function $\psi$ on $\mathbb{R}_{-}$.

The relation between the resolvent and the generator, given in the discrete case by $V_{N, \alpha}^{(n)}=\left(\alpha-A_{N}^{(n)}\right)^{-1}$, and the convergence of the resolvents imply that for every $t>0$, $\int \frac{1}{t-x} d \nu_{N}^{f}(x)$ converges to $\int \frac{1}{t-x} d \nu^{f}(x)$.

By the Stone-Weierstrass theorem, the algebra $\mathcal{A}$ of polynomial functions in $(1-x)^{-1}$ defined on $\mathbb{R}_{-}$is dense in the set $C_{\infty}\left(\mathbb{R}_{-}\right)$of continuous functions on $\mathbb{R}_{-}$vanishing at $-\infty$. Let $\mathcal{C}$ be the vector space spanned by the following set of functions defined on $\mathbb{R}_{-}$, $\left\{x \mapsto \frac{1}{t-x}, t>0\right\}$. As $\mathcal{A}$ is in the closure of $\mathcal{C}$ for the uniform norm, $\mathcal{C}$ is dense in $C_{\infty}\left(\mathbb{R}_{-}\right)$. Thus

converges to

$$
\int f(x) P_{N, t}^{(n)}(f)(x) d m_{N}^{(n)}(x)=\int_{-\infty}^{0} e^{t x} d \nu_{N}^{f}(x)
$$

$$
\int f(x) P_{t}^{(n)}(f)(x) d m^{(n)}(x)=\int_{-\infty}^{0} e^{t x} d \nu^{f}(x)
$$

for all $t>0$. The polarization identity lets us recover the announced convergence.

\subsection{Proof of theorem 8}

First, let us notice that the convergence result stated in proposition 10 also holds if the invariant measures $m_{N}^{(n)}$ and $m^{(n)}$ of the $n$-point processes are replaced by uniform measures:

Proposition 16 Let $n \in \mathbb{N}^{*}$ and $t>0$. Let $\lambda_{N, 0}, \lambda_{N, 1}$ and $\lambda$ denote the uniform probability measures on $\frac{1}{N}(\mathbb{Z} / N \mathbb{Z}), \frac{1}{2 N}((2 \mathbb{Z}+1) / 2 N \mathbb{Z})$ and $S^{1}$ respectively. If $f$ and $g$ are continuous functions on $\left(S^{1}\right)^{n}$ then

$$
\int g(x) P_{N, t}^{(n)}(f)(x) d \lambda_{N, 0}^{\otimes n}(x) \text { and } \int g(x) P_{N, t}^{(n)}(f)(x) d \lambda_{N, 1}^{\otimes n}(x)
$$

converges to $\int g(x) P_{t}^{(n)}(f)(x) d \lambda^{\otimes n}(x)$ as $N$ tends to $+\infty$.

Proof. As $\left\|P_{N, t}^{(n)}(f)\right\|_{\infty} \leq\|f\|_{\infty}$ a density argument reduces the problem to showing the convergence results for $C^{1}$ functions $f$ and $g$. Let $g$ and $f$ be $C^{1}$ functions on $\left(S^{1}\right)^{n}$. First, let us show that $\int g(x) P_{N, t}^{(n)}(f)(x) d \lambda_{N}^{\otimes n}(x)$ converges to $\int g(x) P_{t}^{(n)}(f)(x) d \lambda^{\otimes n}(x)$ as $N$ tends to $+\infty$, where $\lambda_{N}^{(n)}$ denotes the uniform measure on $T_{N}^{(n)}$. For $\varepsilon \geq 0$, set

$$
V_{\varepsilon}=\left\{x \in\left(S^{1}\right)^{n}, \exists i \neq j,\left|x_{i}-x_{j}\right| \leq \varepsilon\right\}
$$

and for $\varepsilon>0$, consider a continuous function $g_{\varepsilon}$ on $\left(S^{1}\right)^{n}$ such that $g_{\varepsilon}=0$ on $V_{0}, g_{\varepsilon}=g$ outside $V_{\varepsilon}$ and $\left\|g_{\varepsilon}\right\|_{\infty} \leq\|g\|_{\infty}$.

Outside $V_{0}$, the measures $m_{N}^{(n)}$ and $m^{(n)}$ coincide with $\lambda_{N}^{(n)}$ and $\lambda^{\otimes n}$ respectively. It follows from proposition 10 that $\int g_{\varepsilon} P_{N, t}^{(n)}(f) d \lambda_{N}^{(n)}$ converges to $\int g_{\varepsilon} P_{t}^{(n)}(f) d \lambda^{\otimes n}$. As $\lambda^{\otimes n}\left(\partial V_{\varepsilon}\right)=0$, $\lambda_{N}^{(n)}\left(V_{\varepsilon}\right)$ converges to $\lambda^{\otimes n}\left(V_{\varepsilon}\right)$. Thus, the upper limit as $N$ tends to $+\infty$ of

$$
\begin{aligned}
\mid \int g P_{N, t}^{(n)}(f) d \lambda_{N}^{(n)} & -\int g P_{t}^{(n)}(f) d \lambda^{\otimes n}\left|\leq \int\right| g-g_{\varepsilon}|| P_{N, t}^{(n)}(f) \mid d \lambda_{N}^{(n)} \\
& +\left|\int g_{\varepsilon} P_{N, t}^{(n)}(f) d \lambda_{N}^{(n)}-\int g_{\varepsilon} P_{t}^{(n)}(f) d \lambda^{\otimes n}\right|+\int\left|g-g_{\varepsilon}\right|\left|P_{t}^{(n)}(f)\right| d \lambda^{\otimes n}
\end{aligned}
$$


is bounded by $4\|g\|_{\infty}\|f\|_{\infty} \lambda^{\otimes n}\left(V_{\varepsilon}\right)$. As $\lambda^{\otimes n}\left(V_{\varepsilon}\right)$ converges to $\lambda^{\otimes n}\left(V_{0}\right)=0$ as $\varepsilon$ tends to 0 , $\left|\int g P_{N, t}^{(n)}(f) d \lambda_{N}^{(n)}-\int g P_{t}^{(n)}(f) d \lambda^{\otimes n}\right|$ converges to 0 as $N$ tends to $+\infty$.

To conclude, let us remark that for every Lipschitz function $\phi$ on $\left(S^{1}\right)^{n}$, the difference between $\int \phi d \lambda_{N}^{(n)}$ and $\int \phi d \lambda_{N, 0}^{\otimes n}$ or $\int \phi d \lambda_{N, 1}^{\otimes n}$ is bounded by

$$
\frac{1}{2 N^{n}} \sum_{u_{1}=0}^{N-1} \cdots \sum_{u_{n}=0}^{N-1}\left|\phi\left(\frac{u_{1}}{N}, \cdots, \frac{u_{n}}{N}\right)-\phi\left(\frac{2 u_{1}+1}{2 N}, \cdots, \frac{2 u_{n}+1}{2 N}\right)\right| \leq \frac{n}{4 N}\|\phi\|_{\text {Lip }} .
$$

As the Lipschitz coefficient of $P_{N, t}^{(n)}(f)$ is bounded by $\|f\|_{\text {Lip }}$, this ends the proof of proposition [16.

\subsubsection{Proof of assertion $(i)$}

Let $t$ be a fixed positive real. We shall prove that for every $j \in \mathbb{N}^{*}$, if $\mu_{N}$ for $N \in \mathbb{N}^{*}$ is a probability measure defined on $\left(\frac{1}{N}(\mathbb{Z} / N \mathbb{Z})\right)^{j}$ such that $\left(\mu_{N}\right)_{N}$ converges weakly to a probability measure $\mu$ on $\left(S^{1}\right)^{j}$ and if $g$ is a continuous function on $\left(S^{1}\right)^{j}$ then the random variables $\mu_{N} K_{N, 0, t}^{\otimes j}(g)$ converge in law towards $\mu K_{0, t}^{\otimes j}(g)$ as $N$ tends to $+\infty$. This convergence result applied to $\mu_{N}=\nu_{N}^{\otimes r}$ and $j=\ell r$ where $r \in \mathbb{N}^{*}$, will show that the measures $E\left(\left(\nu_{N} K_{N, 0, t}^{\otimes \ell}\right)^{\otimes r}\right)$ converge weakly to $E\left(\left(\nu K_{0, t}^{\otimes \ell}\right)^{\otimes r}\right)$. Thus the convergence in law of $\nu_{N} K_{N, 0, t}^{\otimes \ell}$ will follow.

Let $j \in \mathbb{N}^{*}$ and let $\left(\mu_{N}\right)_{N}$ be a sequence of probability measures defined on the sets $\left(\frac{1}{N}(\mathbb{Z} / N \mathbb{Z})\right)^{j}$ that converges weakly to a probability measure $\mu$ on $\left(S^{1}\right)^{j}$. As the $C^{1}$ functions on $\left(S^{1}\right)^{j}$ are dense in $C\left(\left(S^{1}\right)^{j}\right)$, it suffices to prove that for every $g \in C^{1}\left(\left(S^{1}\right)^{j}\right)$, the sequence of random variables $\left(\mu_{N} K_{N, 0, t}^{\otimes j}(g)\right)_{N}$ converges in law to $\mu K_{0, t}^{\otimes j}(g)$. Indeed, let $\left(g_{k}\right)$ be a sequence of $C^{1}$ functions that converges to $g \in C\left(\left(S^{1}\right)^{j}\right)$. For every $u \in \mathbb{R}$,

$$
\begin{aligned}
\left|E\left(e^{i u \mu_{N} K_{N, 0, t}^{\otimes j}(g)}\right)-E\left(e^{i u \mu K_{0, t}^{\otimes j}(g)}\right)\right| & \leq|u| E\left(\left|\mu_{N} K_{N, 0, t}^{\otimes j}\left(g_{k}\right)-\mu_{N} K_{N, 0, t}^{\otimes j}(g)\right|\right) \\
+\mid E\left(e^{i u \mu_{N} K_{N, 0, t}^{\otimes j}\left(g_{k}\right)}\right)- & E\left(e^{i u \mu K_{0, t}^{\otimes j}\left(g_{k}\right)}\right)|+| u \mid E\left(\left|\mu K_{0, t}^{\otimes j}\left(g_{k}\right)-\mu K_{0, t}^{\otimes j}(g)\right|\right) \\
& \leq 2|u||| g-\left.g_{k}\right|_{\infty}+\left|E\left(e^{i u \mu_{N} K_{N, 0, t}^{\otimes j}\left(g_{k}\right)}\right)-E\left(e^{i u \mu K_{0, t}^{\otimes j}\left(g_{k}\right)}\right)\right|
\end{aligned}
$$

Let $\varepsilon>0$. If we take the upper limit of the two parts of the previous inequality as $N$ tends to $+\infty$ with an integer $k$ satisfying $\left\|g-g_{k}\right\|_{\infty} \leq \varepsilon$, then

$$
\varlimsup_{N}\left|E\left(e^{i u \mu_{N} K_{N, 0, t}^{\otimes j}(g)}\right)-E\left(e^{i u \mu K_{0, t}^{\otimes j}(g)}\right)\right| \leq 2|u| \varepsilon
$$

provided that $\mu_{N} K_{N, 0, t}^{\otimes j}(h)$ converges in law to $\mu K_{0, t}^{\otimes j}(h)$ for every $h \in C^{1}\left(\left(S^{1}\right)^{j}\right)$.

Let $g$ be a $C^{1}$ function on $\left(S^{1}\right)^{j}$. In order to prove that $X_{N}=\mu_{N} K_{N, 0, t}^{\otimes j}(g)$ converges in law to $X=\mu K_{0, t}^{\otimes j}(g)$, let us introduce a sequence of probability measures with Lebesgue density that approaches $\mu$. Let $\phi$ be a $C^{\infty}$ density function defined on $\left(S^{1}\right)^{j}$. For $k \in \mathbb{N}^{*}$, set $\phi_{k}: x \mapsto k^{j} \phi(k x)$. Then any probability measure $\eta$ on $\left(S^{1}\right)^{j}$ can be approximated by the probability measures $\left(\phi_{k} \star \eta\right)(x) \lambda^{\otimes j}(d x)$ : more precisely, there is a constant $C_{j, \phi}$ such that for every Lipschitz function $f$ on $\left(S^{1}\right)^{j}$ and every probability measure $\eta$ on $\left(S^{1}\right)^{j}$

$$
\left|\int f\left(\phi_{k} \star \eta\right) d \lambda^{\otimes j}-\int f d \eta\right| \leq \frac{C_{j, \phi}}{k}\|f\|_{\text {Lip }}
$$


Indeed,

$$
\begin{aligned}
& \left|\int f\left(\phi_{k} \star \eta\right) d \lambda^{\otimes j}-\int f d \eta\right| \\
& =\left|\iint f(u) \phi_{k}(u-v) d \lambda^{\otimes j}(u) d \eta(v)-\int f(v)\left(\int \phi_{k}(u-v) d \lambda^{\otimes j}(u)\right) d \eta(v)\right| \\
& \leq\|f\|_{\text {Lip }} \sum_{i=1}^{j} \int\left(\int\left|u_{i}-v_{i}\right| \phi_{k}(u-v) d \lambda^{\otimes j}(u)\right) d \eta(v) .
\end{aligned}
$$

To obtain bound (17), it remains to note that

$$
\int\left|u_{i}-v_{i}\right| \phi_{k}(u-v) d \lambda^{\otimes j}(u)=\frac{1}{k} \int\left|z_{i}\right| \phi(z) d \lambda^{\otimes j}(z) .
$$

Let us introduce auxiliary variables: for $k \in \mathbb{N}^{*}$, set

$$
\begin{aligned}
& X^{(k)}=\int_{\left(S^{1}\right)^{j}} K_{0, t}^{\otimes j}(g)\left(\phi_{k} \star \mu\right) d \lambda^{\otimes j}, X_{N}^{(k)}=\int K_{N, 0, t}^{\otimes j}(g)\left(\phi_{k} \star \mu\right) d \lambda_{N, 0}^{\otimes j}, \\
& Y_{N}^{(k)}=\int K_{N, 0, t}^{\otimes j}(g)\left(\phi_{k} \star \mu_{N}\right) d \lambda_{N, 0}^{\otimes j} .
\end{aligned}
$$

For every $\ell \in \mathbb{N}^{*}$, the $\ell$-th moments of $X^{(k)}$ and $X_{N}^{(k)}$ are the following:

$$
\begin{aligned}
E\left(\left(X_{N}^{(k)}\right)^{\ell}\right) & =\int P_{N, t}^{(j \ell)}\left(g^{\otimes \ell}\right)\left(\phi_{k} \star \mu\right)^{\otimes \ell} d \lambda_{N, 0}^{\otimes \ell j}, \\
E\left(\left(X^{(k)}\right)^{\ell}\right) & =\int P_{t}^{(j \ell)}\left(g^{\otimes \ell}\right)\left(\phi_{k} \star \mu\right)^{\otimes \ell} d \lambda^{\otimes \ell j} .
\end{aligned}
$$

Thus, it follows from proposition [16 that for every $k \in \mathbb{N}^{*}$, the moments of any order of $\left(X_{N}^{(k)}\right)_{N}$ converge to the moments of $X^{(k)}$. As the random variables $X_{N}^{(k)}$ and $X^{(k)}$ are bounded by $\|g\|_{\infty}\left\|\phi_{k}\right\|_{\infty},\left(X_{N}^{(k)}\right)_{N}$ converges in law to $X^{(k)}$. In order to deduce that $\left(X_{N}\right)_{N}$ converges in law to $X$, we shall prove the following results:

- $E\left(\left(X^{(k)}-X\right)^{2}\right)$ converges to 0 as $k$ tends to $+\infty$,

- $\sup _{N \geq k^{j+2}} E\left(\left(Y_{N}^{(k)}-X_{N}\right)^{2}\right)$ converges to 0 as $k$ tends to $+\infty$,

- for every $k \in \mathbb{N}^{*}, E\left(\left(X_{N}^{(k)}-Y_{N}^{(k)}\right)^{2}\right)$ converges to 0 as $N$ tends to $+\infty$.

These three convergence results are sufficient to deduce that the characteristic functions of $X_{N}$ converge pointwise to the characteristic function of $X$. Indeed, for every $u \in \mathbb{R}$ and $k \in \mathbb{N}^{*},\left|E\left(\exp \left(i u X_{N}\right)\right)-E(\exp (i u X))\right|$ is bounded by

$$
\begin{aligned}
&\left|E\left(\exp \left(i u X_{N}\right)\right)-E(\exp (i u X))\right| \leq|u| E\left(\left|X_{N}-Y_{N}^{(k)}\right|\right)+|u| E\left(\left|Y_{N}^{(k)}-X_{N}^{(k)}\right|\right) \\
&+\left|E\left(\exp \left(i u X_{N}^{(k)}\right)\right)-E\left(\exp \left(i u X^{(k)}\right)\right)\right|+|u| E\left(\left|X^{(k)}-X\right|\right) .
\end{aligned}
$$

Thus for every $\varepsilon>0$, there exists an integer $k_{\varepsilon}$ such that for every $N \geq k^{j+2}$,

$$
\begin{aligned}
\left|E\left(\exp \left(i u X_{N}\right)\right)-E(\exp (i u X))\right| \leq|u| \varepsilon+|u| E\left(\left|Y_{N}^{\left(k_{\varepsilon}\right)}-X_{N}^{\left(k_{\varepsilon}\right)}\right|\right) & \\
& +\left|E\left(\exp \left(i u X_{N}^{\left(k_{\varepsilon}\right)}\right)\right)-E\left(\exp \left(i u X^{\left(k_{\varepsilon}\right)}\right)\right)\right| .
\end{aligned}
$$

By taking the upper limit, as $N$ tends to $+\infty$, of the two terms in this inequality, we obtain that $\left|E\left(\exp \left(i u X_{N}\right)\right)-E(\exp (i u X))\right|$ converges to 0 . 
- Study of $E\left(\left(X^{(k)}-X\right)^{2}\right)$ : first,

$$
\begin{aligned}
& E\left(\left(X^{(k)}\right)^{2}-X^{(k)} X\right)= \\
& \int\left(\int P_{t}^{(2 j)}(g \otimes g)(u, v)\left(\phi_{k} \star \mu\right)(u) d \lambda^{\otimes j}(u)\right)\left(\phi_{k} \star \mu\right)(v) d \lambda^{\otimes j}(v) \\
& \quad-\int\left(\int P_{t}^{(2 j)}(g \otimes g)(u, v)\left(\phi_{k} \star \mu\right)(u) d \lambda^{\otimes j}(u)\right) d \mu(v) .
\end{aligned}
$$

As the map $P_{t}^{(2 j)}(g \otimes g)$ is Lipschitz, the map

$$
v \mapsto \int P_{t}^{(2 j)}(g \otimes g)(u, v)\left(\phi_{k} \star \mu\right)(u) d \lambda^{\otimes j}(u)
$$

is also Lipschitz with Lipschitz coefficient bounded by $\left\|P_{t}^{(2 j)}(g \otimes g)\right\|_{\text {Lip }}$. Thus, bound (7) gives $\left|E\left(\left(X^{(k)}\right)^{2}-X^{(k)} X\right)\right| \leq \frac{C_{j, \phi}}{k}\left\|P_{t}^{(2 j)}(g \otimes g)\right\|_{\text {Lip }}$. Similarly,

$$
\begin{aligned}
\left|E\left(X^{(k)} X-X^{2}\right)\right| & =\mid \int\left(\int P_{t}^{(2 j)}(g \otimes g)(u, v) d \mu(u)\right)\left(\phi_{k} \star \mu\right)(v) d \lambda^{\otimes j}(v) \\
- & \int\left(\int P_{t}^{(2 j)}(g \otimes g)(u, v) d \mu(u)\right) d \mu(v) \mid \leq \frac{C_{j, \phi}}{k}\left\|P_{t}^{(2 j)}(g \otimes g)\right\|_{L i p} .
\end{aligned}
$$

Therefore $E\left(\left(X^{(k)}-X\right)^{2}\right)$ converges to 0 as $k$ tends to $+\infty$.

- Study of $E\left(\left(Y_{N}^{(k)}-X_{N}\right)^{2}\right)$ : the same splitting as before yields that

$$
\begin{aligned}
E\left(\left(Y_{N}^{(k)}-X_{N}\right)^{2}\right)=\int\left(F_{N}^{1}(v)-F_{N}^{2}(v)\right)\left(\phi_{k} \star \mu_{N}\right)(v) d \lambda_{N, 0}^{\otimes j}(v) & \\
& -\int\left(F_{N}^{1}(v)-F_{N}^{2}(v)\right) d \mu_{N}(v)
\end{aligned}
$$

where

$$
\begin{aligned}
& F_{N}^{1}(v)=\int P_{N, t}^{(2 j)}(g \otimes g)(u, v)\left(\phi_{k} \star \mu_{N}\right)(u) d \lambda_{N, 0}^{\otimes j}(u) \\
& F_{N}^{2}(v)=\int P_{N, t}^{(2 j)}(g \otimes g)(u, v) d \mu_{N}(u) .
\end{aligned}
$$

Let us bound more generally,

$$
\Delta_{N, k}(f)=\int f\left(\phi_{k} \star \mu_{N}\right) d \lambda_{N, 0}^{\otimes j}-\int f d \mu_{N}
$$

for a Lipschitz function $f$ on $\left(S^{1}\right)^{j} \cdot \Delta_{N, k}(f)$ is the sum of two following terms:

$$
\begin{aligned}
\Delta_{N, k}^{(1)}(f) & =\int f\left(\phi_{k} \star \mu_{N}\right) d \lambda_{N, 0}^{\otimes j}-\int f\left(\phi_{k} \star \mu_{N}\right) d \lambda^{\otimes j} \\
\Delta_{N, k}^{(2)}(f) & =\int f\left(\phi_{k} \star \mu_{N}\right) d \lambda^{\otimes j}-\int f d \mu_{N} .
\end{aligned}
$$


It follows from bound (7) that $\left|\Delta_{N, k}^{(2)}(f)\right| \leq \frac{C_{j, \phi}}{k}\|f\|_{L i p}$. By developing the convolution term $\phi_{k} \star \mu_{N}$, the expression of $\Delta_{N, k}^{(1)}(f)$ becomes

$$
\Delta_{N, k}^{(1)}(f)=\int\left(\int f(u) \phi_{k}(u-v) d \lambda_{N, 0}^{\otimes j}(u)-\int f(u) \phi_{k}(u-v) d \lambda^{\otimes j}(u)\right) d \mu_{N}(v) .
$$

Since for every $v \in\left(S^{1}\right)^{j}, u \mapsto f(u) \phi_{k}(u-v)$ is a Lipschitz function with Lipschitz coefficient bounded by $\|f\|_{L i p} k^{j}\|\phi\|_{\infty}+\|f\|_{\infty} k^{j+1}\|\phi\|_{L i p}$, we obtain

$$
\left|\Delta_{N, k}^{(1)}(f)\right| \leq D_{j} \frac{k^{j+1}}{N}\left(\frac{1}{k}\|f\|_{L i p}\|\phi\|_{\infty}+\|f\|_{\infty}\|\phi\|_{L i p}\right)
$$

where $D_{j}$ is a constant irrespective of $f$ and $\phi$. In conclusion, there is a constant $D_{j, \phi}$ such that for every Lipschitz function $f$ on $\left(S^{1}\right)^{j}$ and $k \in \mathbb{N}^{*}$,

$$
\sup _{N \geq k^{j+2}}\left|\Delta_{N, k}(f)\right| \leq \frac{D_{j, \phi}}{k}\left(\|f\|_{\infty}+\|f\|_{L i p}\right) .
$$

Since the map $P_{N, t}^{(2 j)}(g \otimes g)$ is a Lipschitz function verifying

$$
\left\|P_{N, t}^{(2 j)}(g \otimes g)\right\|_{\infty} \leq\|g \otimes g\|_{\infty} \text { and }\left\|P_{N, t}^{(2 j)}(g \otimes g)\right\|_{L i p} \leq\|g \otimes g\|_{L i p},
$$

$F_{N}^{1}$ and $F_{N}^{2}$ have the same properties. Thus $\sup _{N \geq k^{j+2}} E\left(\left(Y_{N}^{(k)}-X_{N}\right)^{2}\right)$ converges to 0 as $k$ tends to $+\infty$.

- Study of $E\left(\left(Y_{N}^{(k)}-X_{N}^{(k)}\right)^{2}\right)$ : fix an integer $k \in \mathbb{N}^{*}$.

$$
\begin{aligned}
\left|E\left(\left(Y_{N}^{(k)}\right)^{2}-Y_{N}^{(k)} X_{N}^{(k)}\right)\right| & \leq \iint\left(\left|P_{N, t}^{(2 j)}(g \otimes g)(u, v)\right|\left(\phi_{k} \star \mu_{N}\right)(u)\right. \\
\left.\left|\left(\phi_{k} \star \mu_{N}\right)(v)-\left(\phi_{k} \star \mu\right)(v)\right|\right) d \lambda_{N, 0}^{\otimes j}(v) d \lambda_{N, 0}^{\otimes j}(u) & \\
& \leq k^{j}\|g\|_{\infty}^{2}\|\phi\|_{\infty} \int\left|\left(\phi_{k} \star \mu_{N}\right)(v)-\left(\phi_{k} \star \mu\right)(v)\right| d \lambda_{N, 0}^{\otimes j}(v)
\end{aligned}
$$

The term $\left|E\left(\left(X_{N}^{(k)}\right)^{2}-Y_{N}^{(k)} X_{N}^{(k)}\right)\right|$ has the same bound.

The weak convergence of $\left(\mu_{N}\right)_{N}$ to $\mu$ implies that for every $u \in\left(S^{1}\right)^{j}, \phi_{k} \star \mu_{N}(u)$ converges to $\phi_{k} \star \mu(u)$. As for every $N$, the map $\phi_{k} \star \mu_{N}$ is Lipschitz with Lipschitz coefficient bounded by $k^{j+1}\|\phi\|_{L i p},\left(\phi_{k} \star \mu_{N}\right)_{N}$ converges uniformly to $\phi_{k} \star \mu$. It follows that $E\left(\left(Y_{N}^{(k)}-X_{N}^{(k)}\right)^{2}\right)$ converges to 0 as $N$ tends to $+\infty$, for every $k \in \mathbb{N}^{*}$.

\subsubsection{Proof of assertion $(i i)$}

By $(i), \nu_{N} K_{N, 0, t}^{\otimes \ell}(g)$ converges in law to $\nu K_{0, t}^{\otimes \ell}(g)$ as $N$ tends to $+\infty$. These random variables are bounded by $\|g\|_{\infty}$. Thus $E\left(\nu_{N} K_{N, 0, t}^{\otimes \ell}(g)\right)=\int P_{N, 0, t}^{(\ell)}(g) d \nu_{N}$ converges to $E\left(\nu K_{0, t}^{\otimes \ell}(g)\right)=\int P_{0, t}^{(\ell)}(g) d \nu$.

By splitting $f$ into its positive and its negative part, it suffices to deal with a nonnegative function $f$. If $\int f d \nu=0$ then $\left|\int P_{N, t}^{(\ell)}(g) f d \nu_{N}\right| \leq\|g\|_{\infty} \int f d \nu_{N}$ tends to 0. Assume now that $\int f d \nu>0$. Then for $N$ large enough, $\int f d \nu_{N}$ is positive. Let us apply the previous result with the sequence of probability measures $\tilde{\nu}_{N}(d x)=\frac{f(x) d \nu_{N}(x)}{\int f d \nu_{N}}:\left(\tilde{\nu}_{N}\right)$ weakly converges to $\tilde{\nu}(d x)=\frac{f(x) d \nu(x)}{\int f d \nu}$ whence $\frac{1}{\int f d \nu_{N}} \int P_{N, 0, t}^{(\ell)}(g) f d \nu_{N}$ converges to $\frac{1}{\int f d \nu} \int P_{0, t}^{(\ell)}(g) f d \nu$. As $\int f d \nu_{N}$ tends to $\int f d \nu, \int P_{N, 0, t}^{(\ell)}(g) f d \nu_{N}$ converges to $\int P_{0, t}^{(\ell)}(g) f d \nu$. 


\subsubsection{Proof of assertion (iii)}

Set $\mu_{N, t}=\eta_{N} K_{N, 0, t}$ and $\mu_{t}=\eta K_{0, t}$ for every $t \in \mathbb{R}$. Let us prove by iteration that for every $r \in \mathbb{N}^{*}$, the $r$-dimensional distributions of $\mu_{N}$ converge weakly to the $r$-dimensional distributions of $\mu$, that is for every $0=t_{1}<\ldots<t_{r}$, the distribution of $\left(\mu_{N, t_{1}}, \ldots, \mu_{N, t_{r}}\right)$ converges to the distribution of $\left(\mu_{t_{1}}, \ldots, \mu_{t_{r}}\right)$.

First, this convergence result holds for $r=1$. Let $r \in \mathbb{N}^{*}$. Assume that the $r$-dimensional distributions of $\mu_{N}$ converge weakly to those of $\mu$. Let $0=t_{1}<\ldots<t_{r+1}$. To prove that the law of $\left(\mu_{N, t_{1}}, \ldots, \mu_{N, t_{r+1}}\right)$ converges to the distribution of $\left(\mu_{t_{1}}, \ldots, \mu_{t_{r+1}}\right)$, it suffices to show that for every $k_{1}, \ldots, k_{r+1} \in \mathbb{N}$, and for every $g_{1} \in C\left(\left(S^{1}\right)^{k_{1}}\right), \ldots, g_{r+1} \in C\left(\left(S^{1}\right)^{k_{r+1}}\right)$, $E\left(\mu_{N, t_{1}}^{\otimes k_{1}}\left(g_{1}\right) \cdots \mu_{N, t_{r+1}}^{\otimes k_{r+1}}\left(g_{r+1}\right)\right)$ converges to $E\left(\mu_{t_{1}}^{\otimes k_{1}}\left(g_{1}\right) \cdots \mu_{t_{r+1}}^{\otimes k_{r+1}}\left(g_{r+1}\right)\right)$.

First, let us note that for every $N \in \mathbb{N}, \mu_{N, t_{r+1}}$ is equal to $\mu_{N, t_{r}} K_{N, t_{r}, t_{r+1}}$, the random matrix $K_{N, t_{r}, t_{r+1}}$ has the same law as $K_{N, 0, t_{r+1}-t_{r}}$ and is independent of $\left(\mu_{N, t_{1}}, \ldots, \mu_{N, t_{r}}\right)$. Thus,

$$
E\left(\mu_{N, t_{1}}^{\otimes k_{1}}\left(g_{1}\right) \cdots \mu_{N, t_{r+1}}^{\otimes k_{r+1}}\left(g_{r+1}\right)\right)=\int_{\left(S^{1}\right)^{h}} P_{N, t_{r+1}-t_{r}}^{(h)}(G) F d \nu_{N}
$$

where $h=\sum_{i=1}^{r+1} k_{i}, F$ and $G$ are the maps defined on $\left(S^{1}\right)^{h}$ by

$$
F(x, y)=\left(g_{1} \otimes \cdots \otimes g_{r}\right)(x) \text { and } G(x, y)=g_{r+1}(y)
$$

for every $x \in\left(S^{1}\right)^{\sum_{i=1}^{r} k_{i}}$, and $y \in\left(S^{1}\right)^{k_{r+1}}$, and $\nu_{N}$ is the probability measure $E\left(\mu_{N, t_{1}}^{\otimes k_{1}} \otimes \cdots \otimes \mu_{N, t_{r-1}}^{\otimes k_{r-1}} \otimes \mu_{N, t_{r}}^{\otimes\left(k_{r}+k_{r+1}\right)}\right)$.

By the iterative assumption, the distribution of $\left(\mu_{N, t_{1}}, \ldots, \mu_{N, t_{r}}\right)$ converges to the distribution of $\left(\mu_{t_{1}}, \ldots, \mu_{t_{r}}\right)$. Thus in particular, $\left(\nu_{N}\right)_{N}$ weakly converges to $\nu=E\left(\mu_{t_{1}}^{\otimes k_{1}} \otimes \cdots \otimes \mu_{t_{r-1}}^{\otimes k_{r-1}} \otimes \mu_{t_{r}}^{\otimes\left(k_{r}+k_{r+1}\right)}\right)$.

It follows from assertion (ii) that $E\left(\mu_{N, t_{1}}^{\otimes k_{1}}\left(g_{1}\right) \cdots \mu_{N, t_{r+1}}^{\otimes k_{r+1}}\left(g_{r+1}\right)\right)$ converges to $E\left(\mu_{t_{1}}^{\otimes k_{1}}\left(g_{1}\right) \cdots \mu_{t_{r+1}}^{\otimes k_{r+1}}\left(g_{r+1}\right)\right)$, ending the proof of $(i i i)$.

\section{References}

[1] S.N. Ethier and T. G. KuRTz. Markov processes: Characterization and Convergence. Wiley-Interscience, 1986.

[2] M. Fukushima, Y. Oshima, and M. Takeda. Dirichlet forms and symmetric Markov processes. Walter de Gruyter, 1994.

[3] Y. Le Jan and O. Raimond. Flows, coalescence and noise. math.PR/0203221, To appear in The Annals of Probability.

[4] Y. Le JAN and O. Raimond. Sticky flows on the circle and their noises. To appear in Probab. Theory Relat. Fields.

[5] Y. Le JAn and O. RAimond. Sticky flows on the circle. math.PR/0211387, 2002.

[6] E. Pitman. The closest estimates of statistical parameters. Proc. Camb. Philos. Soc., 33:212-222, 1937. 
[7] J. PItman. Some developments of the Blackwell-MacQueen. In L.S. Shapley T.S. Ferguson and J.B. Macqueen, editors, Statistics, Probability and Game Theory, volume 30, pages 245-267. IMS Lecture Notes-Monograph, 1996.

[8] J. Pitman. Combinatorial stochastic processes. Saint-Flour lecture notes, July 2002. 